\title{
Altimeter + INS/Giant LEO Constellation Dual-Satellite Integrated Navigation and Positioning Algorithm Based on Similar Ellipsoid Model and UKF
}

\author{
Lvyang Ye ${ }^{1}\left(\mathbb{D}\right.$, Yikang Yang ${ }^{1, *}$, Xiaolun Jing ${ }^{1}\left(\mathbb{D}\right.$, Hengnian $\mathrm{Li}^{2}$, Haifeng Yang ${ }^{3}$ and Yunxia Xia ${ }^{4,5}$ \\ 1 School of Electronic and Information Engineering, Xi'an Jiao Tong University (XJTU), Xi'an 710049, China; \\ yely2019@stu.xjtu.edu.cn (L.Y.); j2034906@stu.xjtu.edu.cn (X.J.) \\ 2 State Key Laboratory of Astronautic Dynamics, Xi'an Satellite Control Center, Xi'an 710049, China; \\ henry_xscc@mail.xjtu.edu.cn \\ 3 Southwest China Institute of Electronic Technology, Chengdu 610036, China; haifeng_ioe@163.com \\ 4 Institute of Optics and Electronics, Chinese Academy of Sciences, Chengdu 610209, China; \\ xiayunxia@ioe.ac.cn \\ 5 Key Laboratory of Beam Control, Chinese Academy of Sciences, Chengdu 610209, China \\ * Correspondence: yangyk74@mail.xjtu.edu.cn
}

Citation: Ye, L.; Yang, Y.; Jing, X.; Li, H.; Yang, H.; Xia, Y. Altimeter + INS/Giant LEO Constellation Dual-Satellite Integrated Navigation and Positioning Algorithm Based on Similar Ellipsoid Model and UKF. Remote Sens. 2021, 13, 4099. https:// doi.org/10.3390/rs13204099

Academic Editor: Chung-yen Kuo

Received: 7 September 2021

Accepted: 10 October 2021

Published: 13 October 2021

Publisher's Note: MDPI stays neutral with regard to jurisdictional claims in published maps and institutional affiliations.

Copyright: () 2021 by the authors. Licensee MDPI, Basel, Switzerland. This article is an open access article distributed under the terms and conditions of the Creative Commons Attribution (CC BY) license (https:// creativecommons.org/licenses/by/ $4.0 /)$.
Abstract: To solve the problem of location service interruption that is easily caused by incomplete visual satellite environments such as occlusion, urban blocks and mountains, we propose an altimeter + inertial navigation system (INS) + giant low earth orbit (LEO) dual-satellite (LEO2) switching integrated navigation algorithm based on a similar ellipsoid model and unscented Kalman filter (UKF). In addition to effectively improving the INS error, for the INS + LEO dual-satellite switching algorithm without altimeter assistance, our algorithm can also significantly suppress the problem of excessive navigation and positioning error caused by this algorithm in a long switching time, it does not require frequent switching of LEO satellites, and can ensure navigation and positioning functions without affecting LEO satellite communication services. In addition, the vertical dilution of precision (VDOP) value can be improved through the clock error elimination scheme, so, the vertical accuracy can be improved to a certain extent. For different altimeter deviations, we provide simulation experiments under different altimeter deviations; it can be found that after deducting the fixed height deviation, the algorithm can also achieve good accuracy. Compared with other typical algorithms, our proposed algorithm has higher accuracy, lower cost and stronger real-time performance, and is suitable for navigation and positioning scenarios in harsh environments.

Keywords: LEO; altimeter; double star; similar ellipsoid; integrated navigation; switching; navigation and positioning

\section{Introduction}

At present, the global navigation satellite system's (GNSS's) high-precision navigation, positioning and timing functions make it widely used in military, traffic, remote sensing and mapping fields [1-3]. It has many advantages such as global positioning under all-weather conditions, short observation time, and no requirement for intervisibility between observation stations. However, it also has some imperfections, for example, it is easily affected by physical space environmental factors. Moreover, due to the limited number of satellites, communication in harsh environments such as urban blocks, bridges, tunnels, and canyons leads to insufficient GNSS signal strength [4]. In addition, in practical applications, the continuous positioning of the GNSS receiver can not be guaranteed due to satellite failure and carrier attitude change, which leaves the navigation and positioning in an incomplete state. Moreover, to recover from this incomplete state and relocate also consumes a certain amount of time, which is fatal for some real-time applications. 
Compared with the traditional medium earth orbit (MEO) constellation and highly elliptical orbit (HEO) constellation navigation system, the low earth orbit (LEO) constellation satellite has the advantages of fast movement, closer proximity to the earth and low manufacturing cost, such that the LEO constellation has fast positioning convergence, small propagation delay, and rapid deployment. On the one hand, it can be used as a global high-speed data broadcasting channel; since low-orbit communication satellites have larger signal bandwidth and higher information rate, they can be used as the broadcast channel for satellite navigation basic messages and differential correction messages. Since the low orbit constellation is networked around the world, the broadcast range can cover the whole world; when broadcasting basic navigation messages, it can shorten the time to first fix (TTFF) of the receiver cold start, which plays a role similar to assisted global positioning system (AGPS). Additionally, when broadcasting differential correction messages, it can achieve wide-area accuracy enhancement and serve as a precise point positioning (PPP) system. On the other hand, due to the low orbit and fast motion of LEO satellites, the correlation of the observation equations between adjacent epochs is weaker than that of MEO and HEO satellites, so it can also be combined with other types of constellations for fast PPP [5]. In addition, when LEO satellites function as communication satellites, they can eliminate the clock bias between LEO satellites and user receivers through a communication method similar to full-duplex (FD); the accuracy requirements for user clocks are relatively lower, therefore, the preferred constellation scheme is more suitable for the integration of communication and navigation (ICN) [6].

Exploring the navigation enhancement application of LEO constellation. Several studies have discussed the applicability of navigation in low earth orbit (LEO), described the satellites in today's LEO, their role in navigation, the possible LEO constellation configuration in the future, and provided the required mathematical background [7-9]. At the level of constellation design, to overcome the shortcomings of traditional LEO constellations that use monopolar or near-polar orbits for global coverage, which leads to fewer visible satellites at low latitudes, Reference [10] used genetic algorithms to optimize several hybrid LEO enhanced constellations, the algorithm can provide the possibility of $100 \%$ global coverage through one to three LEO satellites used for broadband internet access. A new algorithm is used that is based on the LEO constellation, using a differential carrier phase measurement architecture which can effectively solve the integer ambiguity solution [11], but the algorithm is similar to the MEO constellation-based integer ambiguity solution algorithm. Reference [12] gave a virtual wideband (VWB) receiver architecture, discussed the applicability of the signal provided by the PNT service on the giant LEO constellation, and analyzed the Doppler upper limit level that could be tolerated, however, although VWB can tolerate harsh Doppler conditions and reduce the required processing bandwidth, LEO has a limited time to pass the zenith; long-term band occupation will waste band resources and cause unstable frequency sources, and the error increases with time.

Aiming at the difficulty of navigation and positioning caused by the lack of visible satellites. References $[13,14]$ designed a low-order Kalman filter for the BeiDou double-star positioning system, and the successive approximation method was used to solve the problem of position lag in the BeiDou double-star system. The actual sports car verification was carried out, however, the low order filter will bring some problems, such as phase lag, low sensitivity and poor smoothing effect, which will affect the final navigation and positioning effect. To overcome the shortcomings of the BeiDou dual-satellite navigation and positioning system with active positioning, a new passive positioning algorithm was developed that was based on the data collected by two synchronous satellites, as well as the atomic clock and altimeter carried by the user and through a grid search which realized passive positioning [15]. However, this algorithm required the assistance of an atomic clock, which undoubtedly increases the size, power consumption and cost of the device. Reference [16] was given an elevation model of the BeiDou double-star navigation and positioning system and combined with two BeiDou satellites for positioning; the simulation results proved that the algorithm had the characteristics of fast convergence and accurate positioning. 
The algorithm can be regarded as a typical traditional double star + altimeter navigation and positioning algorithm; the application in some challenging environments will be restricted. Reference [17] proposed a dual-satellite positioning algorithm to improve the positioning performance under the satellite signal gap; the algorithm used the previously stored positioning data under complete navigation conditions to simplify the positioning equation, since the clock deviation lasts for a short time, so, the method requires only two visible satellites to obtain accurate positioning results without calculating clock deviations, but the premise of the algorithm is to store navigation data under complete conditions, and requires high clock stability, it is known that the algorithm will also be affected by navigation lag and face the problem of high equipment cost. Aiming at the problem that the GNSS receiver cannot achieve positioning due to insufficient available satellites under incomplete conditions, a navigation and positioning algorithm based on multi-epoch double-differential pseudorange observation (MDPO) is proposed, which uses multi-epoch observations to reduce the number of navigation satellites required [18]; obviously, this algorithm requires multiple observations to achieve positioning solution.

The solution is aimed at the situation where global positioning system (GPS) signals may be accidentally shielded in complex and challenging environments such as obstructions, urban blocks and mountains. Reference [19] considered accessing the stronger signal transmitted from the LEO communication satellite system to assist GPS services; the algorithm used the Doppler frequency shift as the measurement value and developed a positioning algorithm based on Doppler measurement, but the algorithm can only be used as an enhanced system to assist GPS in navigation and positioning. Reference [20] proposed a comprehensive navigation algorithm based on a giant low earth orbit (LEO) constellation; the algorithm realizes alternate switching of ranging by introducing a real ranging value and a virtual ranging value. That approach is different from the traditional dual-satellite integrated navigation algorithm in that it can complete accurate real-time navigation and positioning without the aid of an altimeter and continuous observation, however, in this algorithm, when the switching time is relatively large, the positioning error may deteriorate, especially when switching is based on the same orbit. Reference [21] proposed a combined navigation and positioning algorithm based on the relative motion information measured by the INS, the position information of the two satellites and the pseudorange information at multiple times under the scenario of only 2 visible satellites; this algorithm can ensure stable, reliable navigation and positioning results, similarly, the algorithm also needs to observe through continuous position information.

Our article makes full use of the many advantages of the LEO constellation, and considers using it as a tentative study of a navigation and positioning scheme for the whole system rather than an augmented system. When only two visible LEO satellites are available, the proposed algorithm can effectively complete the precise navigation and positioning service by alternately switching, and combining with the altimeter under the establishment of a reasonable elevation measurement model. In Section 2 of the article, on the basis of the principle of altimeter height measurement, based on the concept of similar ellipsoids, the observations related to user elevation are established, and derivation is carried out we then describe the basic principle and process of the algorithm in detail. In Section 3, we established the state equations and measurement equations of the entire system. In Section 4, by setting the simulation conditions and parameters reasonably, simulation experiments were performed on different scenarios and the qualitative and quantitative analysis results were given; at the same time, our algorithm was compared with other typical algorithms. Our conclusions are given in the last section of the article.

\section{Algorithm Principle}

The principle of the algorithm is similar to that in Reference [20], and the difference is that we have considered the altimeter and established a reasonable elevation model. For the convenience of analysis, we will briefly restate the algorithm described in Reference [20]; similarly, we will also perform calculations regardless of the clock bias-the clock bias was 
eliminated by an FD method in default, and the improvement of the vertical dilution of precision (VDOP) brought about by the clock bias elimination is analyzed. At the same time, we also use strap-down INS and the WGS-84 Earth-centered Earth-Fixed (ECEF) coordinate system for analysis and modeling.

\subsection{The Principle of Barometric Height Measurement and Its Auxiliary Positioning Algorithm}

2.1.1. The Principle of Barometric Pressure Measurement

Generally, the information obtained by measuring air pressure can assist the GNSS navigation and positioning system to increase the availability of the corresponding system [22]. The positioning accuracy of GNSS system in the vertical direction is relatively poor, especially in areas with large topographic relief, such as a winding road section or for high building structures, however, with the help of an altimeter, we can measure the relative height of the moving carrier, and effectively improve the accuracy of the system in the elevation direction. Common altimeters include a barometric altimeter and a radar altimeter; here, we use a barometric altimeter for analysis.

In practical engineering applications, the barometric altimeter measures the height of the moving carrier, the change of local atmospheric pressure, and the relative height that changes due to geographical changes based on the law of atmospheric pressure changes. We know that the air pressure will decrease as the height increases. According to this law, we can use the following formula to determine the height information of the moving carrier [23]:

$$
H_{k}=\frac{T_{t}}{\nabla_{t}}\left[\left(\frac{P_{0}}{P_{S}}\right)^{-\left(\frac{R_{d} \nabla_{t}}{g_{0}}\right)}-1\right]
$$

where $T_{t}$ is the surface temperature, $\nabla_{t}=6.5 \times 10^{-3} \mathrm{~K} \mathrm{~m}^{-1}$ is the atmospheric temperature gradient, $P_{0}$ is the atmospheric pressure at height $\mathrm{z}, P_{S}=1013,25 \mathrm{~h} \mathrm{~Pa}, R_{d}=278.1 \mathrm{~J} \cdot \mathrm{kg}^{-1} \cdot \mathrm{K}^{-1}$ is the gas constant (under dry air conditions), and $g_{0}=9.80665 \mathrm{~m} \cdot \mathrm{s}^{-2}$.

2.1.2. Clock Bias Elimination and Altimeter-Assisted Positioning Algorithm Based on Similar Ellipsoid

(1) Elevation information model based on similar ellipsoid

According to the WGS84 coordinate system, we regard the earth as an ellipsoid, and we assume that the moving carrier is located on an ellipsoid that coincides with the center of the earth model, the semi-major axis (equatorial radius), semi-minor axis (polar radius), and the radii of the unitary circle coincide, and the eccentricity is equal; we call the earth (small ellipsoid) and the large ellipsoid similar ellipsoids. Suppose that the true position of the ECEF coordinate of the carrier on the large ellipsoid is $\left(x_{c}, y_{c}, z_{c}\right)$, and the reference ECEF coordinate position on the earth (small ellipsoid) is $\left(x^{\prime}{ }_{c}, y^{\prime}{ }_{c}, z^{\prime}{ }_{c}\right)$; to use the related properties of similar ellipsoids, we first prove that the two ellipsoids are similar.

Definition 1. Ellipsoids with coincident centers, long semi axis (equatorial radius), short semi axis (polar radius), overlapping radius of prime unitary circle and equal eccentricity are similar ellipsoids.

Theorem 1. Assume that the semi-major axes of the small ellipsoid (Earth) and the large ellipsoid are: $R_{e}, R_{E}$, and the semi-minor axes are: $R_{p}, R_{P}$, and the radii of the circle at the same latitude are: $R_{S}, R_{L}$, which is shown in Figure 1. If a large ellipsoid is similar to a small ellipsoid, it should have:

$$
\frac{R_{E}}{R_{e}}=\frac{R_{P}}{R_{p}}=\frac{R_{L}}{R_{S}}
$$




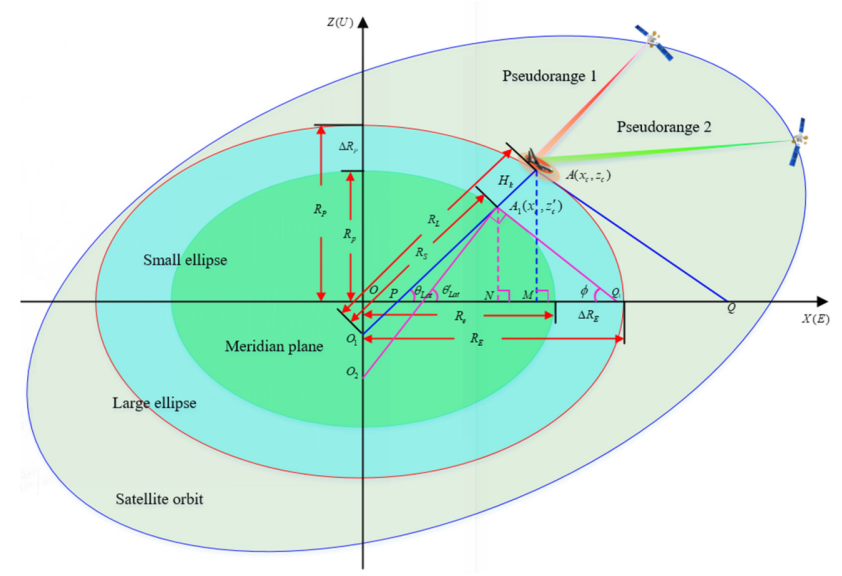

Figure 1. 2-dimensional schematic diagram of similar ellipsoid.

Proof: Let the spatial geodetic coordinate system (WGS-84) be attached to the reference ellipsoid. Since the reference ellipsoid is symmetrical around the $z$-axis, we can convert the three-dimensional ellipsoid into a two-dimensional plane rectangular coordinate system for analysis.

From the definition:

$$
e^{2}=\frac{R_{E}^{2}-R_{P}^{2}}{R_{E}^{2}}=\frac{R_{e}^{2}-R_{p}^{2}}{R_{e}^{2}}
$$

So we can get:

$$
\frac{R_{E}}{R_{e}}=\frac{R_{P}}{R_{p}} .
$$

Since the moving carrier is located at point $\mathrm{A}$ on the large ellipsoid and the plane coordinate is $\left(x_{c}, z_{c}\right)$, the slope of the straight line $\mathrm{AO}_{1}$ is [16]:

$$
\tan \theta_{\text {Lat }}=\frac{R_{E}^{2}}{R_{P}^{2}} \cdot \frac{z}{x} .
$$

We cross point $\mathrm{A}_{1}$ to make the normal line of the small ellipse and the polar axis intersects at point $\mathrm{O}_{2}$, the angle between the straight line $\mathrm{A}_{1} \mathrm{O}_{2}$ and the $x$-axis is $\theta_{\text {Lat }}^{\prime}$, and now cross point $\mathrm{A}_{1}$ to make the tangent of the small ellipse and the $x$-axis intersect at point $Q_{1}$, and the angle with the $x$-axis is $\phi$. The slope of the tangent $A_{1} Q_{1}$ is [16]:

$$
-\tan \phi=-\tan \left(\frac{\pi}{2}-\theta_{\text {Lat }}^{\prime}\right)=-\cot \theta_{\text {Lat }}^{\prime}=-\frac{R_{p}^{2}}{R_{e}^{2}} \cdot \frac{x_{c}^{\prime}}{z_{c}^{\prime}}=-\frac{1}{\frac{R_{e}^{2}}{R_{p}^{2}} \cdot \frac{z_{c}^{\prime}}{x^{\prime}}} .
$$

We use $\mathrm{A}$ and $\mathrm{A}_{1}$, respectively, to make the vertical line of the $x$-axis and cross the $x$-axis at the two points of $M$ and $N$. According to the principle of triangle similarity, we can obtain $\triangle \mathrm{APM} \sim \Delta \mathrm{A}_{1} \mathrm{PN}$, then:

$$
\frac{x_{c}}{z_{c}}=\frac{x_{c}^{\prime}}{z_{c}^{\prime}}
$$

Then from Equations (4)-(7), we can obtain:

$$
\tan \theta_{\text {Lat }} \cdot \tan \phi=-1 .
$$

From the Formula (8), it can be seen that $\mathrm{A}_{1} Q_{1}$ and the straight line $\mathrm{AO}_{1}$ are perpendicular to each other. Furthermore, we cross point $A$ to make the tangent of the large ellipse and the $x$-axis intersect at point $Q$; then from Equation (8) and its conclusion, we can see 
that the straight line $\mathrm{A}_{1} Q_{1}$ is parallel to the straight line $\mathrm{AQ}$, that is to say, the straight lines of the primary unitary circle radii passing through points $\mathrm{A}_{1}$ and $\mathrm{A}$ are coincident.

According to the formula of the primary unitary circle radius of the small ellipse and the large ellipse at latitude $\theta_{\text {Lat }}$, we can obtain [24]:

$$
\left\{\begin{array}{c}
R_{S}=\frac{R_{e}}{\sqrt{1-e^{2} \sin ^{2} \theta_{\text {Lat }}}} \\
R_{L}=\frac{R_{E}}{\sqrt{1-e^{2} \sin ^{2} \theta_{\text {Lat }}}}=R_{e}+H_{k}
\end{array} .\right.
$$

Then there are:

$$
\frac{R_{L}}{R_{S}}=\frac{R_{E}}{R_{e}}=\frac{R_{S}+H_{k}}{R_{S}} .
$$

From Equations (4) and (10), we can obtain: $\frac{R_{E}}{R_{e}}=\frac{R_{P}}{R_{p}}=\frac{R_{L}}{R_{S}}$; at this point, the proof process is complete.

Since the height of the moving carrier is changing, the large ellipsoid also changes momentarily, but this does not affect the relative properties of similar ellipsoids. According to the point $\mathrm{A}\left(x_{c}, y_{c}, z_{c}\right)$ on the large ellipsoid where the moving carrier is located and the measured height information is $H_{k}$, an ellipsoid equation can be approximately established [25]:

$$
\frac{x_{c}^{2}}{\rho_{0 k}^{2}}+\frac{y_{c}^{2}}{\rho_{0 k}^{2}}+\frac{z_{c}^{2}}{\rho_{P k}^{2}}=1,
$$

where $\rho_{0 k}=R_{e}+H_{k}, \rho_{P k}=R_{p}+H_{k}, R_{e}=6378.137 \mathrm{~km}$, and $R_{p}=6356.752 \mathrm{~km}$.

In addition, since the clock bias between the receiver carried by the moving carrier and the LEO satellite was considered to be completely eliminated, that is, we do not need to consider the solution of the clock bias at this time, we only need the pseudorange measurement information of the two satellites in order to complete the positioning solution.

(2) The improvement principle of VDOP by elimination of clock bias

When the clock bias between the receiver on the moving carrier and the LEO satellites is eliminated by exploiting the two-way communication link [6], we assume that the coordinates of each LEO satellite in the ECEF coordinate system are $\left(x_{i}, y_{i}, z_{i}\right)$, and $\rho_{i L E O}=\sqrt{\left(x_{i}-x_{c}\right)^{2}+\left(y_{i}-y_{c}\right)^{2}+\left(z_{i}-z_{c}\right)^{2}}$ represents the pseudorange measurement value to the $i$-th satellite. We omit the complicated derivation and directly give the corresponding geometric matrix as follows:

$$
H^{\dagger}=\left[\begin{array}{lll}
\frac{\partial \rho_{1 L E O}}{\partial x_{c}} & \frac{\partial \rho_{1 L E O}}{\partial y_{c}} & \frac{\partial \rho_{1 L E O}}{\partial z_{c}} \\
\frac{\partial \rho_{2 L E O}}{\partial x_{c}} & \frac{\partial \rho_{2 L E O}}{\partial y_{c}} & \frac{\partial \rho_{2 L E O}}{\partial z_{c}} \\
\frac{\partial \rho_{3 L E O}}{\partial x_{c}} & \frac{\partial \rho_{3 L E O}}{\partial y_{c}} & \frac{\partial \rho_{3 L E O}}{\partial z_{c}}
\end{array}\right],
$$

where there are:

$$
\left\{\begin{array}{l}
\frac{\partial \rho_{i L E O}}{\partial x_{c}}=\frac{\left(x_{c}-x_{i}\right)}{\rho_{i L E O}} \mid x_{c}=x_{\Theta} \\
\frac{\partial \rho_{i L E O}}{\partial y_{c}}=\frac{\left(y_{c}-y_{i}\right)}{\rho_{i L E O}} \mid y_{c}=y_{\Theta} \\
\frac{\partial \rho_{i L E O}}{\partial z_{c}}=\frac{\left(z_{c}-z_{i}\right)}{\rho_{i L E O}} \mid z_{c}=z_{\Theta}
\end{array},\right.
$$

and where $\left(x_{\Theta}, y_{\Theta}, z_{\Theta}\right)$ is the nominal position solution of $\left(x_{c}, y_{c}, z_{c}\right)$.

The covariance matrix of the position becomes:

$$
\operatorname{cov}(\text { Pos })=\left[\begin{array}{ccc}
\sigma_{x_{c}}^{2} & \sigma_{x_{c} y_{c}}^{2} & \sigma_{x_{c} z_{c}}^{2} \\
\sigma_{x_{c} y_{c}}^{2} & \sigma_{y_{c}}^{2} & \sigma_{y_{c} z_{c}}^{2} \\
\sigma_{x_{c} z_{c}}^{2} & \sigma_{y_{c} z_{c}}^{2} & \sigma_{z_{c}}^{2}
\end{array}\right] \text {. }
$$




$$
\begin{aligned}
& \text { Let } H^{\Xi}=\left[\left(H^{\dagger}\right)^{T} H^{\dagger}\right]^{-1} \text {, then: } \\
& \operatorname{cov}(\text { Pos })=\left[\begin{array}{ccc}
H_{x x}^{\Xi} & H_{x y}^{\Xi} & H_{x z}^{\Xi} \\
H_{x y}^{\Xi} & H_{y y}^{\Xi} & H_{y z}^{\Xi} \\
H_{x z}^{\Xi} & H_{y z}^{\Xi} & H_{z z}^{\Xi}
\end{array}\right] \sigma_{U R E}^{2},
\end{aligned}
$$

where $\sigma_{U R E}^{2}$ is the variance of the user ranging error (URE), and $\sigma_{x_{c^{\prime}}}^{2} \sigma_{y_{c}}^{2}$, and $\sigma_{z_{c}}^{2}$ are the variances of the corresponding components of each positioning error, respectively. Finally, VDOP can be expressed as:

$$
V D O P=\frac{\sigma_{z_{c}}^{2}}{\sigma_{U R E}^{2}}=\sqrt{H_{z z}^{\Xi}} .
$$

It can be seen that when the clock bias is eliminated, the covariance matrix is independent of the clock state; at this time, VDOP will become smaller, since the precision factor can be regarded as a linear mapping from the measurement error in the observation to the state estimation error. In the case of the same measurement error, a smaller precision factor will make the error of the state estimation smaller; this is important for improving the VDOP value, because the receiver cannot receive evenly distributed signals from the satellite at the zenith position and it is impossible to receive signals from the other side of the earth.

\subsection{Introduction to UKF Algorithm and Its Implementation Steps}

In actual engineering applications, the systems we encounter are often non-linear systems, and the use of standard Kalman filtering often cannot strictly meet the required conditions. Therefore, under normal circumstances, we can solve practical problems through the extended Kalman filter Jacobian (EKF) algorithm. However, in essence, the EKF algorithm is a linearization method for nonlinear estimation problems. It needs to obtain the analytical form of the system to calculate the Jacobian matrix. When encountering strong nonlinear problems, the estimation accuracy is significantly reduced. To overcome the shortcomings of the EKF algorithm and to avoid the calculation of the Jacobian matrix, which is computationally intensive and error-prone, Julier et al. proposed an unscented Kalman filter (UKF) algorithm based on an unscented transformation (UT) transformation [26,27]. The UKF algorithm approximates the probability density distribution of the nonlinear function, and uses a series of determined samples to approximate the posterior probability density of the state, instead of approximating the nonlinear function, and does not need to derive to calculate the Jacobian matrix. The UKF algorithm does not linearize and ignores higher-order terms, so the accuracy of the nonlinear sub-statistics is higher. Based on this, we use the UKF algorithm for filtering estimation in the article. The specific solving steps of the UKF algorithm are as follows:

We consider the following nonlinear system:

$$
\left\{\begin{array}{l}
X_{k+1}=f\left(X_{k}, \theta_{k}\right) \\
Y_{k}=h\left(X_{k}\right)+v_{k}
\end{array} .\right.
$$

where, $X_{k}$ is the state variable of the system, $Y_{k}$ is the observation vector, $\theta_{k}$ is the process noise of the system, and $v_{k}$ is the measurement noise. We assumed that the process noise and the measurement noise have Gaussian distributions, and there is no correlation between the two. Under this assumption, we can obtain the UKF filtering algorithm design process as follows:

(1) Initialization

For Equation (17), the augmented state vector $\hat{X}^{*}$ and state covariance matrix $D^{*}$ are used: 
(1) State initialization

$$
\left\{\begin{array}{l}
\hat{X}_{0}=E\left\{X_{0}\right\} \\
D_{0}=E\left\{\left(X_{0}-\hat{X}_{0}\right)\left(X_{0}-\hat{X}_{0}\right)^{\mathrm{T}}\right\}
\end{array} .\right.
$$

(2) Expanded dimension processing

$$
\left\{\begin{array}{l}
\hat{X}_{0}^{*}=E\left\{X_{0}^{*}\right\}=\left[\begin{array}{c}
\hat{X}_{0} \\
O_{m \times 1}
\end{array}\right] \\
D_{0}^{*}=E\left\{\left(X_{0}^{*}-\hat{X}_{0}^{*}\right)\left(X_{0}^{*}-\hat{X}_{0}^{*}\right)^{\mathrm{T}}\right\}=\left[\begin{array}{ccc}
D_{0} & 0 & 0 \\
0 & Q & 0 \\
0 & 0 & R
\end{array}\right]
\end{array}\right.
$$

where $m$ is the dimensionality of the system process noise, $O$ is the zero matrix, and the Sigma point set $X_{i}(i=0,1, \cdots, 2 L)$ is as follows:

$$
\left\{\begin{array}{ll}
X_{0}=\bar{X} & \\
X_{i}=\bar{X}+\left(\sqrt{(L+\mu) D_{X}}\right)_{i}, & i=1,2, \cdots, L \\
X_{i}=\bar{X}-\left(\sqrt{(L+\mu) D_{X}}\right)_{i}, & i=L+1, L+2, \cdots, 2 L
\end{array} .\right.
$$

where $X$ is an $L$-dimensional random variable that satisfies the nonlinear equation $Y=g(X)$. We assume that the mean of the variable $X$ is $\bar{X}$; the variance of $X$ is $D_{X} ;\left(\sqrt{(L+\mu) D_{X}}\right)_{i}$ represents the $i$-th row of the square root of $(L+\mu) D_{X}$; $\mu=\alpha^{2}(L+k)-L$, where, $\alpha$ describes the extent of the Sigma point around the mean; and $k$ is the adjustment parameter.

(2) Calculate the sampling point

Combining Equation (20), using mean $\hat{X}_{k-1}^{*}$ and variance $D_{k-1}^{*}$, calculate $2 L+1$

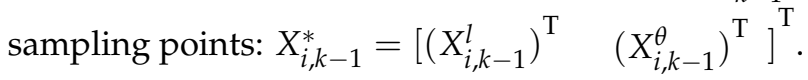

$$
\left\{\begin{array}{l}
X_{0, k-1}^{*}=\hat{X}_{k-1}^{*} \\
X_{i, k-1}^{*}=\hat{X}_{k-1}^{*}+\left(\sqrt{(L+\mu) D_{k-1}^{*}}\right)_{i}, \quad i=1,2, \cdots, L \\
X_{i, k-1}^{*}=\hat{X}_{k-1}^{*}-\left(\sqrt{(L+\mu) D_{k-1}^{*}}\right)_{i-L^{\prime}} \quad i=L+1, L+2, \cdots, 2 L
\end{array} .\right.
$$

(3) Time update

(1) State one-step prediction matrix:

$$
X_{i, k / k-1}^{l}=f\left(X_{i, k-1}^{l}, X_{i, k-1}^{\theta}\right), \quad i=0,1, \cdots, 2 L .
$$

(2) State mean weighted processing:

$$
\hat{X}_{k}^{-}=\sum_{i=0}^{2 L} \kappa_{i}^{(n)} X_{i, k / k-1}^{l} .
$$

where, $\kappa_{i}(i=0,1, \cdots, 2 L)$ is the weight corresponding to the Sigma point set $X_{i}$ :

$$
\left\{\begin{array}{l}
\kappa_{0}^{(n)}=\mu /(L+\mu) \\
\kappa_{0}^{(j)}=\mu /(L+\mu)+\left(1-\alpha^{2}+\beta\right) \\
\kappa_{i}^{(n)}=\kappa_{i}^{(j)}=1 /(2(L+1)), \quad i=1,2, \cdots, 2 L
\end{array}\right.
$$

where $\beta$ describes the system distribution information. To determine the mean and variance of $Y$, we can transform $Y=g(X)$ to get point $Y_{i}=g\left(X_{i}\right)$, and calculate 
the mean and variance of $Y_{i}$ according to the obtained point $Y$ and the corresponding weights:

$$
\left\{\begin{array}{l}
\bar{Y}=\sum_{i=0}^{2 L} \kappa_{i}^{(n)} Y_{i} \\
D_{Y}=\sum_{i=0}^{2 L} \kappa_{i}^{(j)}\left(Y_{i}-\bar{Y}\right)\left(Y_{i}-\bar{Y}\right)^{\mathrm{T}}
\end{array} .\right.
$$

(3) State variance weighted processing:

$$
D_{k}^{-}=\sum_{i=0}^{2 L} \kappa_{i}^{(j)}\left(X_{i, k / k-1}^{l}-\hat{X}_{k}^{-}\right)\left(X_{i, k / k-1}^{l}-\hat{X}_{k}^{-}\right)^{\mathrm{T}} .
$$

(4) One-step observation equation of state:

$$
Y_{i, k / k-1}=h\left(X_{i, k / k-1}^{l}\right), \quad i=0,1, \cdots, 2 L .
$$

(5) Observation weighting processing:

$$
\hat{Y}_{k}^{-}=\sum_{i=0}^{2 L} \kappa_{i}^{(n)} Y_{i, k / k-1} .
$$

(4) Measurement update

(1) Covariance matrix weighting processing of the observations:

$$
D_{Y_{k} Y_{k}}=\sum_{i=0}^{2 L} \kappa_{i}^{(j)}\left(Y_{i, k / k-1}-\hat{Y}_{k}^{-}\right)\left(Y_{i, k / k-1}-\hat{Y}_{k}^{-}\right)^{\mathrm{T}} .
$$

(2) Covariance matrix weighting processing of the state value and observation value:

$$
D_{X_{k} Y_{k}}=\sum_{i=0}^{2 L} \kappa_{i}^{(j)}\left(X_{i, k / k-1}^{l}-\hat{X}_{k}^{-}\right)\left(Y_{i, k / k-1}-\hat{Y}_{k}^{-}\right)^{\mathrm{T}} .
$$

(3) Filter gain matrix:

$$
K_{U K F}=D_{X_{k} Y_{k}} D_{Y_{k} Y_{k}}^{-1}
$$

(4) State estimation equation:

$$
\hat{X}_{k}=\hat{X}_{k}^{-}+K_{U K F}\left(Y_{k}-\hat{Y}_{k}^{-}\right) .
$$

(5) Estimated error variance matrix:

$$
D_{k}=D_{k}^{-}-K_{U K F} D_{Y_{k} Y_{k}} K_{U K F}{ }^{\mathrm{T}} .
$$

We can see from the calculation process of the UKF filter that the UKF filter does not need to be linearized, which avoids the introduction of linearization errors, thereby improving the estimation accuracy. In addition, the UKF filter simplifies greatly the implementation effort; it is precisely because of this that the UKF filter is widely used in the field of navigation and positioning [28].

\subsection{Standard INS + LEO Dual-Satellite (LEO2) + Altimeter Integrated Navigation and Positioning}

The principle of the standard INS + LEO dual-satellite (LEO2) switching + altimeter integrated navigation algorithm is: First, we use satellite ephemeris data stored by broadband LEO satellites. The position of the satellite in the orbital coordinate system can be calculated, and then converted to the ECEF coordinate system, so as to obtain 
the coordinate position of the satellite in the ECEF coordinate system. Then the satellite position of the adjacent time can be differentiated to obtain the satellite's velocity. Then use the position and velocity information which is provided by the INS, and combine with data from an altimeter of the carrier, thereby we can obtain the elevation information. Then use the information to calculate the distance and distance rate relative to the position and speed of the INS, respectively. Subsequently, we determine the difference of the above measured values, and then use them as the filtered observations of UKF, so as to obtain the best estimation value of the LEO satellite and INS error. Finally, the errors of LEO satellite and INS systems are calibrated, respectively. The schematic diagram is shown in Figure 2.

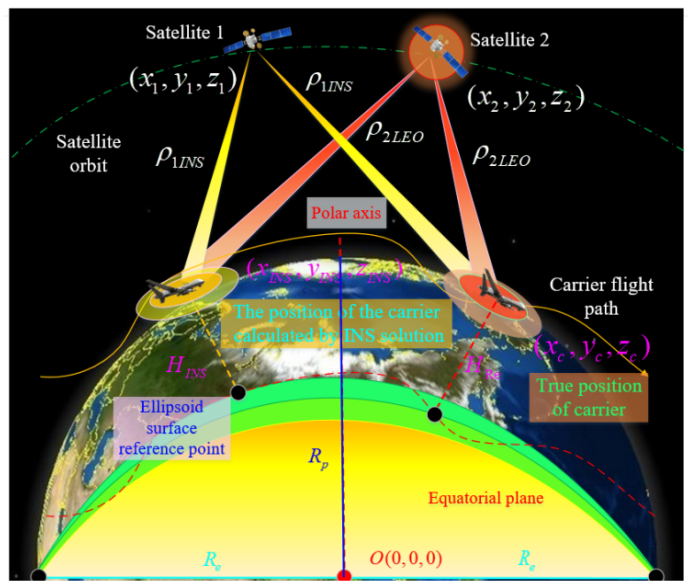

Figure 2. Standard INS + LEO2 + altimeter integrated navigation and positioning principle diagram.

According to the description in Figure 2, we assume that the ECEF coordinate of satellite 1 is $\left(x_{1}, y_{1}, z_{1}\right)$, the ECEF coordinate of satellite 2 is $\left(x_{2}, y_{2}, z_{2}\right)$, and the moving carrier coordinate obtained by the INS solution is $\left(x_{I N S}, y_{I N S}, z_{I N S}\right) . \rho_{1 L E O}$ and $\rho_{2 L E O}$ represent the actual ranging values of satellite 1 and satellite 2 to the moving carrier's true distance obtained by satellite 1 and satellite 2 in real time through the ephemeris, respectively; $\rho_{1 I N S}$ and $\rho_{2 I N S}$ represent the distance between the current satellite position calculated by satellite 1 and satellite 2 through the ephemeris to the moving carrier position obtained by the INS solution, respectively. $H_{I N S}$ is the height value of the carrier position given by the INS, and $H_{\text {Re }}$ is the height value measured by the altimeter of the moving carrier receiver. Based on this, we can list the following equations:

$$
\left\{\begin{array}{l}
\rho_{1 L E O}=\sqrt{\left(x_{1}-x_{c}\right)^{2}+\left(y_{1}-y_{c}\right)^{2}+\left(z_{1}-z_{c}\right)^{2}} \\
\rho_{2 L E O}=\sqrt{\left(x_{2}-x_{c}\right)^{2}+\left(y_{2}-y_{c}\right)^{2}+\left(z_{2}-z_{c}\right)^{2}} \\
\rho_{1 I N S}=\sqrt{\left(x_{1}-x_{I N S}\right)^{2}+\left(y_{1}-y_{I N S}\right)^{2}+\left(z_{1}-z_{I N S}\right)^{2}} \\
\rho_{2 I N S}=\sqrt{\left(x_{2}-x_{I N S}\right)^{2}+\left(y_{2}-y_{I N S}\right)^{2}+\left(z_{2}-z_{I N S}\right)^{2}} \\
\frac{x_{c}^{2}}{\rho_{0-I N S}^{2}}+\frac{y_{c}^{2}}{\rho_{0-I N S}^{2}}+\frac{z_{c}^{2}}{\rho_{\mathrm{P}-I N S}^{2}}=1 \\
\frac{x_{c}^{2}}{\rho_{0-R e}^{2}}+\frac{y_{c}^{2}}{\rho_{0-R e}^{2}}+\frac{z_{c}^{2}}{\rho_{\mathrm{P}-\mathrm{Re}}^{2}}=1
\end{array}\right.
$$

where $\rho_{0-I N S}=R_{e}+H_{I N S}, \rho_{\mathrm{P}-I N S}=R_{p}+H_{I N S}, \rho_{0-R e}=R_{e}+H_{\mathrm{Re}}, \rho_{\mathrm{P}-\mathrm{Re}}=R_{p}+H_{\mathrm{Re}}$, regardless of $H_{I N S}$ or $H_{\mathrm{Re}}$, which can be determined from the standard atmosphere model Formula (1). 
According to Equation (17), we calculate the difference between the first term and the third term, the second term and the fourth term, and the last two terms of the equation, respectively; we then obtain:

$$
\left\{\begin{array}{l}
\hat{\rho}_{1-I N S}=\rho_{1 L E O}-\rho_{1 I N S} \\
\hat{\rho}_{2-I N S}=\rho_{2 L E O}-\rho_{2 I N S} \\
\rho_{0}=\left(\frac{1}{\rho_{0-R e}^{2}}-\frac{1}{\rho_{0-I N S}^{2}}\right) /\left(\frac{1}{\rho_{\mathrm{P}-\mathrm{Re}}^{2}}-\frac{1}{\rho_{\mathrm{P}-I N S}^{2}}\right)=\frac{z_{c}^{2}}{x_{c}^{2}+y_{c}^{2}}
\end{array} .\right.
$$

Then use $\hat{\rho}_{1-I N S}, \hat{\rho}_{2-I N S}$ and $\rho_{0}=\left(\frac{1}{\rho_{0-\mathrm{Re}}^{2}}-\frac{1}{\rho_{0-I N S}^{2}}\right) /\left(\frac{1}{\rho_{\mathrm{P}-\mathrm{Re}}^{2}}-\frac{1}{\rho_{\mathrm{P}-I N S}^{2}}\right)$ as the filtering observations of the system algorithm, and then through the UKF filtering algorithm, finally, the three-dimensional position of the moving carrier is solved.

\subsection{INS + LEO2 Alternate Switching + Altimeter Integrated Navigation Algorithm}

\subsubsection{Algorithm Description}

According to the minimum geometric dilution precision (GDOP) satellite selection algorithm [6,29], two LEO satellites whose positions are visible at any time during the flight of the moving carrier are selected, and we temporarily name them LEO-1 and LEO-2. Similarly, we assume that the ECEF coordinates of the moving carrier measured by INS extrapolation are $\left(\breve{x}_{c}, \breve{y}_{c}, \breve{z}_{c}\right) . \breve{\rho}_{1}$ and $\breve{\rho}_{2}$ represent the measurements between the satellite position calculated by satellite 1 and satellite 2 through the ephemeris to the moving carrier position measured by INS extrapolation in real time, respectively, since they are not actual measurements, so we call them virtual ranging values [20]. Subsequently, the two satellites alternately switch periodically with the real ranging value and the virtual ranging value, and the switching period is $\Delta t$. The schematic diagram is shown in Figure 3.

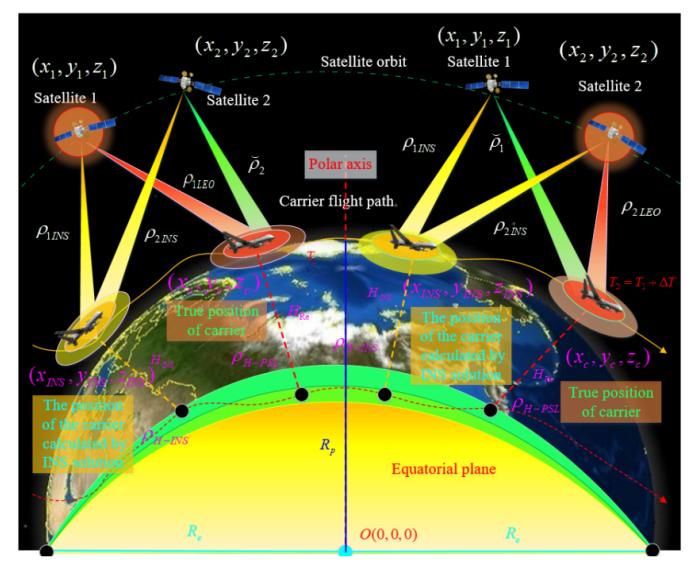

Figure 3. INS + LEO2 alternate switching + altimeter integrated navigation algorithm principle diagram.

\subsubsection{Algorithm Processing Flow}

According to Figure 3 , at time $T_{1}$, let LEO-1 use the real ranging value $\rho_{1 L E O}$ and LEO-2 use the virtual ranging value $\breve{\rho}_{2}$. At this time, we can obtain:

$$
\left\{\begin{array}{l}
\rho_{1 L E O}=\sqrt{\left(x_{1}-x_{c}\right)^{2}+\left(y_{1}-y_{c}\right)^{2}+\left(z_{1}-z_{c}\right)^{2}} \\
\breve{\rho}_{2}=\sqrt{\left(x_{2}-\breve{x}_{c}\right)^{2}+\left(y_{2}-\breve{y}_{c}\right)^{2}+\left(z_{2}-\breve{z}_{c}\right)^{2}} \\
\rho_{1 I N S}=\sqrt{\left(x_{1}-x_{I N S}\right)^{2}+\left(y_{1}-y_{I N S}\right)^{2}+\left(z_{1}-z_{I N S}\right)^{2}} \\
\rho_{2 I N S}=\sqrt{\left(x_{2}-x_{I N S}\right)^{2}+\left(y_{2}-y_{I N S}\right)^{2}+\left(z_{2}-z_{I N S}\right)^{2}} \\
\frac{x_{c}^{2}}{\rho_{0-I N S}^{2}}+\frac{y_{c}^{2}}{\rho_{0-I N S}^{2}}+\frac{z_{c}^{2}}{\rho_{\mathrm{P}-I N S}^{2}}=1 \\
\frac{x_{c}^{2}}{\rho_{0-R e}^{2}}+\frac{y_{c}^{2}}{\rho_{0-R e}^{2}}+\frac{z_{c}^{2}}{\rho_{\mathrm{P}-\mathrm{Re}}^{2}}=1
\end{array}\right.
$$


Similar to the processing of Formula (17), we use the following difference of Formula (19) as the filtering observation of the system algorithm.

$$
\left\{\begin{array}{l}
\hat{\rho}_{1-I N S}=\rho_{1 L E O}-\rho_{1 I N S} \\
\hat{\rho}_{\breve{2}-I N S}=\breve{\rho}_{2}-\rho_{2 I N S} \\
\rho_{0}=\left(\frac{1}{\rho_{0-R e}^{2}}-\frac{1}{\rho_{0-I N S}^{2}}\right) /\left(\frac{1}{\rho_{\mathrm{P}-\mathrm{Re}}^{2}}-\frac{1}{\rho_{\mathrm{P}-I N S}^{2}}\right)
\end{array} .\right.
$$

At time $T_{2}=T_{1}+\Delta T$, let LEO- 1 use the virtual ranging value $\breve{\rho}_{1}$ and let LEO-2 use the real ranging value $\rho_{2 L E O}$. At this time, we can obtain:

$$
\left\{\begin{array}{l}
\breve{\rho}_{1}=\sqrt{\left(x_{1}-\breve{x}_{c}\right)^{2}+\left(y_{1}-\breve{y}_{c}\right)^{2}+\left(z_{1}-\breve{z}_{c}\right)^{2}} \\
\rho_{2 L E O}=\sqrt{\left(x_{2}-x_{c}\right)^{2}+\left(y_{2}-y_{c}\right)^{2}+\left(z_{2}-z_{c}\right)^{2}} \\
\rho_{1 I N S}=\sqrt{\left(x_{1}-x_{I N S}\right)^{2}+\left(y_{1}-y_{I N S}\right)^{2}+\left(z_{1}-z_{I N S}\right)^{2}} \\
\rho_{2 I N S}=\sqrt{\left(x_{2}-x_{I N S}\right)^{2}+\left(y_{2}-y_{I N S}\right)^{2}+\left(z_{2}-z_{I N S}\right)^{2}} \\
\frac{x_{c}^{2}}{\rho_{0-I N S}^{2}}+\frac{y_{c}^{2}}{\rho_{0-I N S}^{2}}+\frac{z_{c}^{2}}{\rho_{\mathrm{P}-I N S}^{2}}=1 \\
\frac{x_{c}^{2}}{\rho_{0-R e}^{2}}+\frac{y_{c}^{2}}{\rho_{0-R e}^{2}}+\frac{z_{c}^{2}}{\rho_{\mathrm{P}-\mathrm{Re}}^{2}}=1
\end{array}\right.
$$

In the same way, we use the following difference in Equation (21) as the filtering observation of the system algorithm.

$$
\left\{\begin{array}{l}
\hat{\rho}_{\breve{1}-I N S}=\breve{\rho}_{1}-\rho_{1 I N S} \\
\hat{\rho}_{2-I N S}=\rho_{2 L E O}-\rho_{2 I N S} \\
\rho_{0}=\left(\frac{1}{\rho_{0-R e}^{2}}-\frac{1}{\rho_{0-I N S}^{2}}\right) /\left(\frac{1}{\rho_{\mathrm{P}-\mathrm{Re}}^{2}}-\frac{1}{\rho_{\mathrm{P}-I N S}^{2}}\right)
\end{array} .\right.
$$

From now on, this alternate switching process will be repeated, that is, every $\Delta T$ time, the real and virtual ranging value of LEO-1 and LEO-2 are switched, until the end of the whole flight test time of the moving carrier.

\section{Establishment of Integrated Navigation Model}

3.1. State Equation and Measurement Equation

\subsubsection{INS State Equation}

The error state equation of INS can be described as [6]:

$$
\dot{U}_{I N S}(t)=A_{I N S}(t) U_{I N S}(t)+B_{I N S}(t) V_{I N S}(t),
$$

where the state variable $U_{I N S}(t)$ is as follows:

$$
U_{I N S}=\left[\begin{array}{llllll}
\Phi_{1 \times 3} & V_{1 \times 3} & \Lambda_{1 \times 3} & \psi_{1 \times 3} & \Gamma_{1 \times 3}
\end{array}\right]^{T},
$$

in the formula $\Phi_{1 \times 3}=\left[\varphi_{E}, \varphi_{N}, \varphi_{U}\right]$ represents the attitude matrix of the moving carrier's roll angle, pitch angle and yaw angle, respectively; and $\Lambda_{1 \times 3}=[\delta L, \delta \lambda, \delta h]$ represents the moving carrier's latitude, longitude and altitude error matrix, respectively. $V_{1 \times 3}=\left[\delta V_{E}, \delta V_{N}, \delta V_{U}\right]$ represents the velocity error matrix; $\psi_{1 \times 3}=\left[\psi_{E}, \psi_{N}, \psi_{U}\right]$ represents the first-order Markov shift matrix of the gyroscope; $\Gamma_{1 \times 3}=\left[\Gamma_{E}, \Gamma_{N}, \Gamma_{U}\right]$ is the first-order Markov shift matrix of the accelerometer; and E, $\mathrm{N}$ and $\mathrm{U}$ represent the east, north and up directions of the east-north-up coordinate system, respectively.

The process noise matrix is:

$$
V_{I N S}(t)=\left[\begin{array}{cc}
\omega_{1 \times 3} & \omega_{1 \times 3}
\end{array}\right]^{T},
$$

where $\omega_{1 \times 3}=\left[\omega_{x}, \omega_{y}, \omega_{z}\right]$ is the random drift noise matrix of the accelerometer, and $\omega_{1 \times 3}=\left[\omega_{x}, \omega_{y}, \omega_{z}\right]$ is the random drift noise matrix of the gyroscope. 
$A_{I N S}(t) \in \mathbb{R}^{15 \times 15}$ and $B_{I N S}(t) \in \mathbb{R}^{15 \times 6}$ are the state transition matrix and the noise driving matrix, respectively.

\subsubsection{LEO's Equation of State}

The state equation of the giant LEO system can be described as [20]:

$$
\dot{U}_{L E O}(t)=B_{L E O}(t) V_{L E O}(t)
$$

where $V_{L E O}(t)=\left[\begin{array}{c}\sigma_{t u} \\ \sigma_{t r u}\end{array}\right], B_{L E O}(t)=\left[\begin{array}{ll}1 & 0 \\ 0 & 1\end{array}\right]$, and $\sigma_{t u}$ and $\sigma_{t r u}$ are Gaussian white noise with mean value of zero.

Combining Formulas (11) and (23), finally, the state equation of the whole combined system is as follows:

$$
\left[\begin{array}{c}
\dot{U}_{I N S}(t) \\
\dot{U}_{L E O}(t)
\end{array}\right]=A_{I N S}(t) U_{I N S}(t)+\left[\begin{array}{cc}
B_{I N S}(t) & 0 \\
0 & B_{L E O}(t)
\end{array}\right]\left[\begin{array}{c}
V_{I N S}(t) \\
V_{L E O}(t)
\end{array}\right] .
$$

\subsection{Measurement Equation of Integrated Navigation System}

The observation equations of INS and LEO based on the pseudorange and pseudorange rate are as follows [6]:

$$
\left\{\begin{array}{l}
Y_{\rho}=R_{\rho} X+W_{\rho} \\
\dot{Y}_{\rho}=\dot{R}_{\rho} X+\dot{W}_{\rho}
\end{array}\right.
$$

After sorting, the measurement equations for the tight combination of INS and LEO can be obtained as follows:

$$
Y(t)=R(t) X(t)+W(t)=\left[\begin{array}{c}
Y_{\rho}(t) \\
\dot{Y}_{\rho}(t)
\end{array}\right]=\left[\begin{array}{c}
R_{\rho}(t) \\
\dot{R}_{\rho}(t)
\end{array}\right] X(t)+\left[\begin{array}{c}
W_{\rho}(t) \\
\dot{W}_{\rho}(t)
\end{array}\right]
$$

where $R_{\rho}=\left[O_{N \times 6} R_{\rho 1} O_{N \times 6} R_{\rho 2}\right], \dot{R}_{\rho}=\left[O_{N \times 3} \dot{R}_{\rho 1} O_{N \times 9} \dot{R}_{\rho 2}\right], N$ is the number of satellites, $W_{\rho}=\left[\begin{array}{lll}W_{\rho 1} & W_{\rho 2} & W_{\rho 3}\end{array}\right]^{T}$ and $\dot{W}_{\rho}=\left[\begin{array}{lll}\dot{W}_{\rho 1} & \dot{W}_{\rho 2} & \dot{W}_{\rho 3}\end{array}\right]^{T}$ are white noise matrices; and

$$
\left\{\begin{array}{l}
R_{\rho 1}=\left[\begin{array}{lll}
\alpha_{i x_{c}} & \alpha_{i y_{c}} & \alpha_{i z_{c}}
\end{array}\right]_{3 \times 3} \delta_{L \lambda h}^{E C E F} \\
\dot{R}_{\rho 1}=\left[\begin{array}{lll}
\alpha_{i x_{c}} & \alpha_{i y_{c}} & \alpha_{i z_{c}}
\end{array}\right]_{3 \times 3} \delta_{E N U}^{E C E F} \quad,(i=1,2,3), \\
R_{\rho 2}=\left[\begin{array}{ll}
-1 & 0 \\
\dot{R}_{\rho 2} & =\left[\begin{array}{ll}
-1 & 0
\end{array}\right]
\end{array}\right.
\end{array}\right.
$$

where $\alpha_{i x_{u}}=\frac{x_{c}-x_{L E O}^{i}}{\rho_{i}}, \alpha_{i y_{u}}=\frac{y_{\mathcal{c}}-y_{L E O}^{i}}{\rho_{i}}, \alpha_{i z_{u}}=\frac{z_{\mathcal{c}}-z_{L E O}^{i}}{\rho_{i}} ; \rho_{i}$ represents the actual distance between the moving carrier and the corresponding LEO satellite, $i$ represents the $i$-th satellite, $\left(x_{L E O}^{i}, y_{L E O}^{i}, z_{L E O}^{i}\right)$ is the LEO satellite position calculated by ephemeris; $\delta_{L \lambda h}^{E C E F}$ represents the coordinate conversion matrix from longitude, latitude and altitude to the ECEF coordinate system, and $\delta_{E N U}^{E C E F}$ represents the coordinate conversion matrix from the ENU coordinate system to the ECEF coordinate system.

\subsection{Other Error Models}

Other error models for the INS and LEO satellites, such as the gyro error, accelerometer error, ephemeris error, ionospheric and tropospheric error models can be found in the References [24,30,31]. For interference models, such as line-of-sight (LOS)/non-line-of-sight (NLOS), multipath interference model, noise and other interference models can refer to the References [20,32-37]. Here, our noise interference model is consistent with the Gaussian noise interference model adopted in Reference [20]; in addition, due to the complexity of multipath modeling, we will not consider it here. 


\section{Simulation Experiment}

\subsection{System and Initial Parameter Settings}

\subsubsection{Experimental Parameters}

For the system simulation, we take the SpaceX core constellation; the flight trajectory is composed of level flight, turning, climbing, and acceleration, and the main parameters are shown in Tables 1 and 2 [38,39].

Table 1. Main parameters of the system.

\begin{tabular}{ll}
\hline Orbital Parameters & Value \\
\hline Cycle $(\mathrm{min})$ & 120 \\
Height $(\mathrm{km})$ & 1150 \\
Orbit radius $(\mathrm{km})$ & 7521 \\
Inclination $\left(^{\circ}\right)$ & 53 \\
Track surface number & 32 \\
Number of satellites on a single orbital surface & 50 \\
Total number of satellites & 1600 \\
\hline
\end{tabular}

Table 2. Initial parameter setting of orbit.

\begin{tabular}{ll}
\hline Parameter Type & Value \\
\hline Initialization position error & $(0 \mathrm{~m}, 0 \mathrm{~m}, 0 \mathrm{~m}) \mathrm{ENU}$ \\
Initialization velocity error & $(0 \mathrm{~m} / \mathrm{s}, 0 \mathrm{~m} / \mathrm{s}, 0 \mathrm{~m} / \mathrm{s}) \mathrm{ENU}$ \\
Initialization attitude error & $\left(0^{\circ}, 0^{\circ}, 0^{\circ}\right) \mathrm{ENU}$ \\
Initial position & $\left(50.425^{\circ} \mathrm{N},-3.5958^{\circ} \mathrm{E}, 10,000 \mathrm{~m}\right)$ \\
Initializing velocity & $200 \mathrm{~m} / \mathrm{s}$ \\
Initial attitude & $0^{\circ} /$ Roll, $0^{\circ} /$ Pitch, $90^{\circ} /$ Yaw \\
\hline
\end{tabular}

\subsubsection{Experimental Situation}

The experiment selects the best two visible satellites LEO-1 and LEO-2 during the flight of the carrier according to the GOP selection satellite algorithm, and the corresponding PRN number is given, respectively. Due to the low orbit of the LEO satellite, according to Kepler's law of motion, it can be known that the LEO satellite moves very fast, which will lead to a very short time for the LEO satellite to pass the zenith, therefore, we must switch between satellites and we set the switching time to 5, 10, 30, 60 and 90 s, respectively [20]. In addition, to explore the navigation and positioning performance of the algorithm under different altimeter deviations, so as to provide a reference basis for practical engineering application, we divide the altimeter deviation into three scenarios: no deviation, fixed deviation $\pm 5 \mathrm{~m}$ and fixed deviation $\pm 20 \mathrm{~m}$. See Table 3 for all parameters.

Table 3. The main parameter settings of the experimental situation.

\begin{tabular}{ll}
\hline Parameter & Value \\
\hline Satellite elevation & $10^{\circ}$ \\
Same orbit experimental satellite & LEO-1:PRN $=233, \mathrm{LEO}-2: \mathrm{PRN}=245$ \\
Adjacent orbit experimental satellite & LEO-1:PRN $=245, \mathrm{LEO}-2: \mathrm{PRN}=269$ \\
Switching time $\Delta t$ & $5,10,30,60,90 \mathrm{~s}$ \\
Altimeter deviation & No deviation, fixed deviation $\pm 5 \mathrm{~m}$, fixed \\
& deviation $\pm 20 \mathrm{~m}$ \\
\hline
\end{tabular}

\subsection{Experimental Results}

4.2.1. INS + LEO2 + Unbiased Altimeter and No Altimeter Scenario Experiment under the Same Orbit

According to the parameter settings in Tables 1-3, in the same orbit, the experimental results of the INS + LEO2 switching + unbiased altimeter algorithm are shown in Figure 4. 
At the same time, the experiment of the INS + LEO2 switching without an altimeter algorithm under the same orbit is also given. As a comparison, we included the standard INS + LEO2 switching navigation and positioning algorithm and the standard INS + LEO2 switching + altimeter navigation and positioning algorithm. Where, INS means pure INS navigation algorithm; the standard INS + two LEO satellites alternate switching ranging algorithm; we abbreviate it as: Standard INS + LEO2. Similarly, for the standard INS + two LEO satellites alternate switching ranging + altimeter algorithm, we abbreviate it as: Standard INS + LEO2 + Altimeter; unbiased altimeter $+\Delta t \mathrm{~s}$ switching algorithm of two LEO satellites, we abbreviate it as: unbiased altimeter $+\Delta t \mathrm{~s}$ switching, the value of $\Delta t$ is shown in Table 3; and the alternate switching algorithm of two LEO satellites without the aid of an altimeter is abbreviated as $\Delta t \mathrm{~s}$ switching. In addition, the meaning of each $y$-axis title is as follows, EPE: east position error; NPE: north position error; UPE: up position error, the meaning of velocity error is similar, while in attitude error, AEE: attitude error of east, other abbreviated meanings by analogy, there is no special description in the following diagrams, and all can be understood according to the above abbreviations.
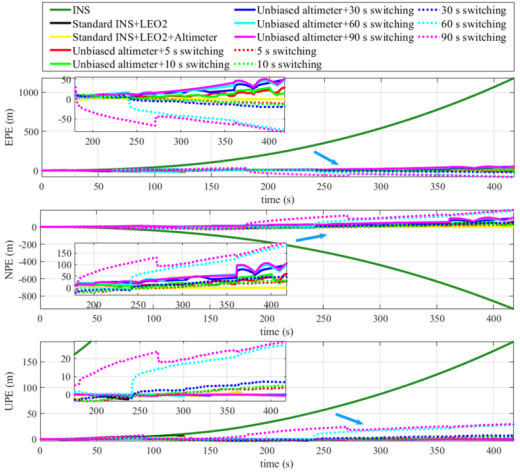

(a) Position error curve

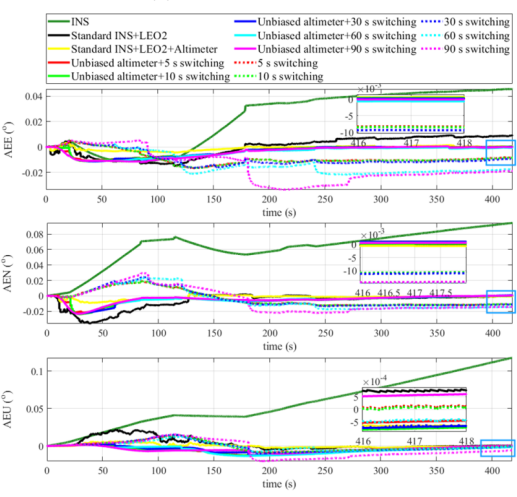

(c) Attitude error curve

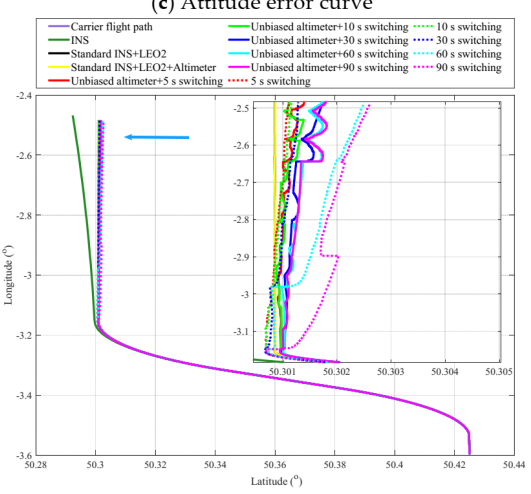

(e) 2-dimensional trajectory error curve

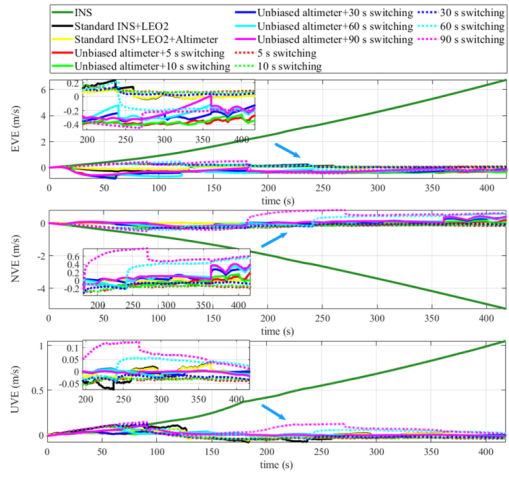

(b) Velocity error curve

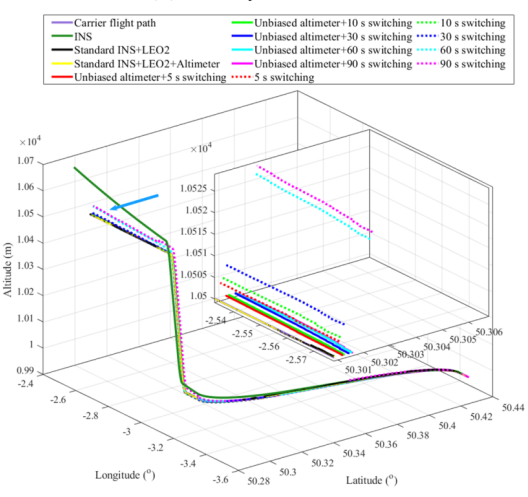

(d) 3-dimensional trajectory error curve

Figure 4. Experimental results of INS + LEO2 + unbiased altimeter and no altimeter scenarios under the same orbit. 
From Figure 4, we can see that the INS + LEO2 switching + unbiased altimeter navigation and positioning algorithm can also effectively suppress the divergence of INS. Compared to the INS + LEO2 switching algorithm without an altimeter, after combining the altimeter, the algorithm can effectively suppress the divergence of various errors under relatively large switching times (such as 60 and 90 s). In this way, the large switching time divergence can be suppressed without frequent switching of LEO satellites, which can ensure the navigation and positioning functions without affecting the communication services of the LEO satellites. We count the final trajectory errors of the larger switching times of 60 and $90 \mathrm{~s}$, as shown in Figure 5.

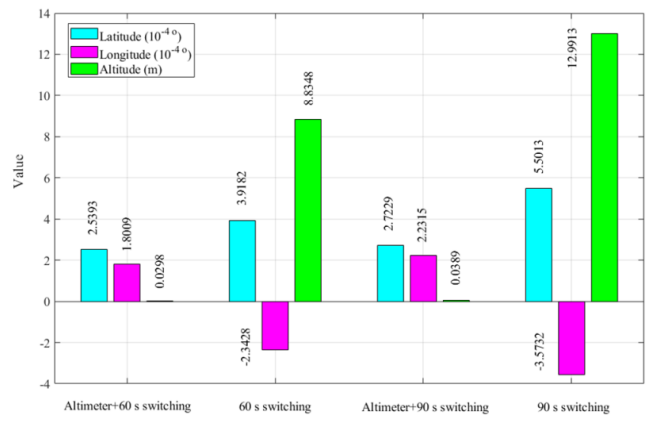

(a) Mean value statistics

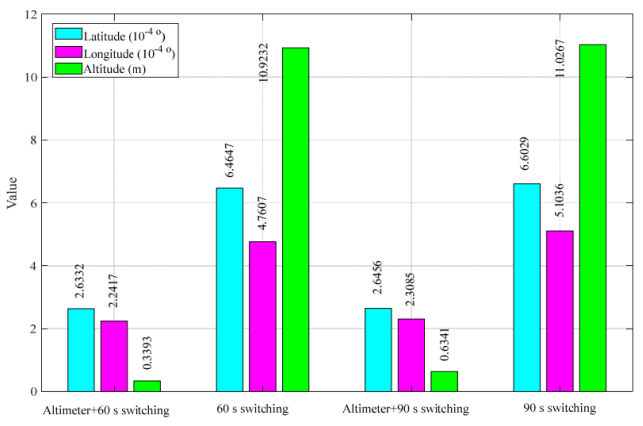

(b) Standard deviation statistics

Figure 5. Under the same orbit, the navigation and positioning error statistics of the INS + LEO2 switching + unbiased altimeter algorithm and the INS + LEO2 switching algorithm when the switching time is 60 and $90 \mathrm{~s}$, respectively.

From Figure 5, we can obtain that with the assistance of the altimeter, when the switching time is $60 \mathrm{~s}$, comparing with the INS + LEO2 switching algorithm, the INS + LEO2 switching + unbiased altimeter navigation and positioning algorithm in longitude, latitude and altitude's errors improve by $35.19 \%, 23.13 \%$ and $99.66 \%$, respectively, and the corresponding standard deviation performance improve by $59.27 \%, 52.91 \%$ and $96.89 \%$, respectively. When the switching time is $90 \mathrm{~s}$, comparing with the INS + LEO2 switching algorithm, the INS + LEO2 switching + unbiased altimeter navigation and positioning algorithm in longitude, latitude and altitude's errors improve by $50.50 \%, 37.55 \%$, and $99.70 \%$, respectively, and the corresponding standard deviation performance values improve by $59.93 \%, 54.77 \%$ and $94.25 \%$, respectively. It can be seen that due to the addition of the altimeter, the new algorithm has the most obvious improvement in height error, this result is consistent with the analysis result in Section 2.1.2, and we will not analyze it in detail here.

4.2.2. INS + LEO2 + Unbiased Altimeter and No Altimeter Scenario Experiment under Adjacent Tracks

According to the parameter settings in Tables 1-3, in adjacent orbits, the experimental results of the INS + LEO2 switching + unbiased altimeter algorithm and the INS + LEO2 switching algorithm in adjacent orbits are shown in Figure 6. Similarly, as a comparison, we also included the standard INS + LEO2 switching navigation and positioning algorithm, and the standard INS + LEO2 switching + altimeter navigation and positioning algorithm as a reference.

From Figure 6 we can see that, in addition to effectively suppressing the divergence of INS, the INS + LEO2 + unbiased altimeter algorithm in adjacent orbits can also suppress the error divergence problem of the INS + LEO2 switching algorithm under a longer switching time, especially when the switching time is $90 \mathrm{~s}$. The final trajectory error statistical results of the two algorithms at 60 and $90 \mathrm{~s}$ are shown in Figure 7.

From Figure 7 we can obtain that, with the aid of the altimeter, when the switching time is $60 \mathrm{~s}$, comparing with the INS + LEO2 switching algorithm, the INS + LEO2 switching + unbiased altimeter navigation and positioning algorithm in longitude, latitude and 
altitude's errors improve by $3.13 \%, 49.45 \%$ and $97.58 \%$, respectively, and the corresponding standard deviations improve by $43.27 \%, 31.85 \%$ and $92.21 \%$, respectively. When the switching time is $90 \mathrm{~s}$, comparing with the INS + LEO2 switching algorithm, the INS + LEO2 switching + unbiased altimeter navigation and positioning algorithm in longitude, latitude and altitude's errors improve by $59.88 \%, 47.96 \%$ and $99.58 \%$, respectively, and the corresponding standard deviation performances improve by $74.00 \%, 60.22 \%$ and $93.41 \%$, respectively. It can be found that the addition of an altimeter also improves the height error considerably, and the principle is the same as that under the same orbit.
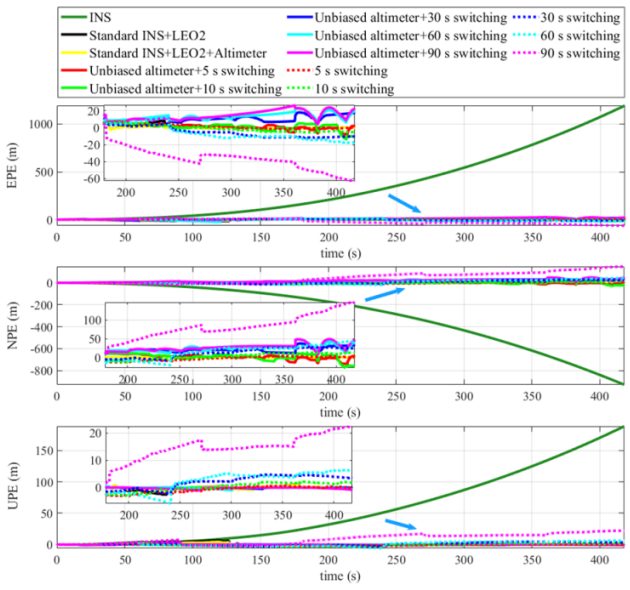

(a) Position error curve
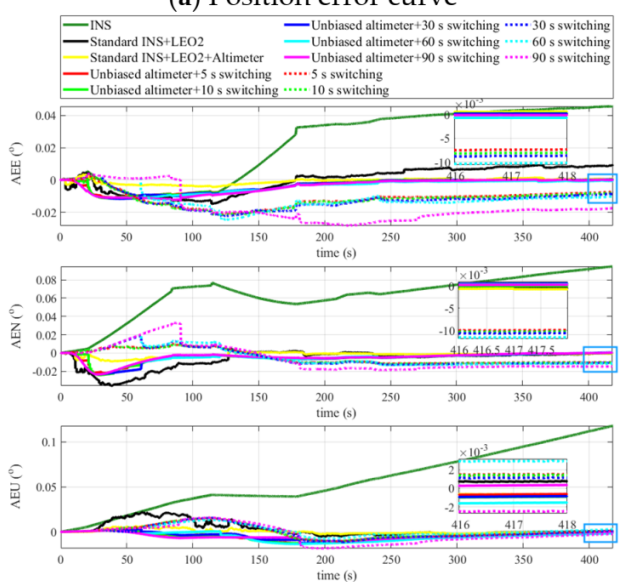

(c) Attitude error curve

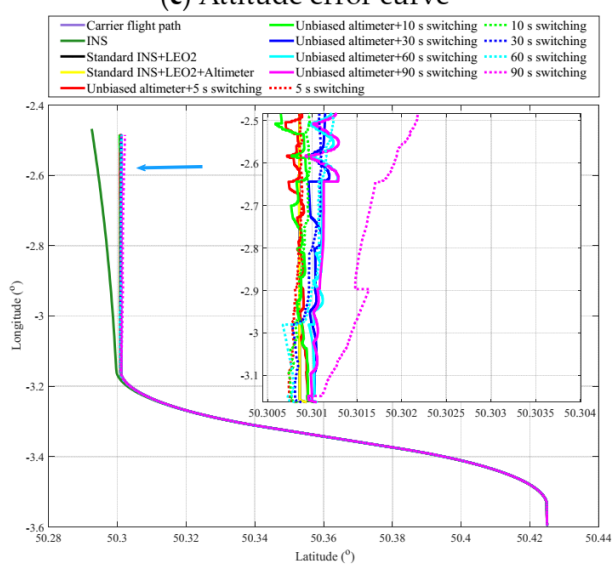

(e) 2-dimensional trajectory error curve

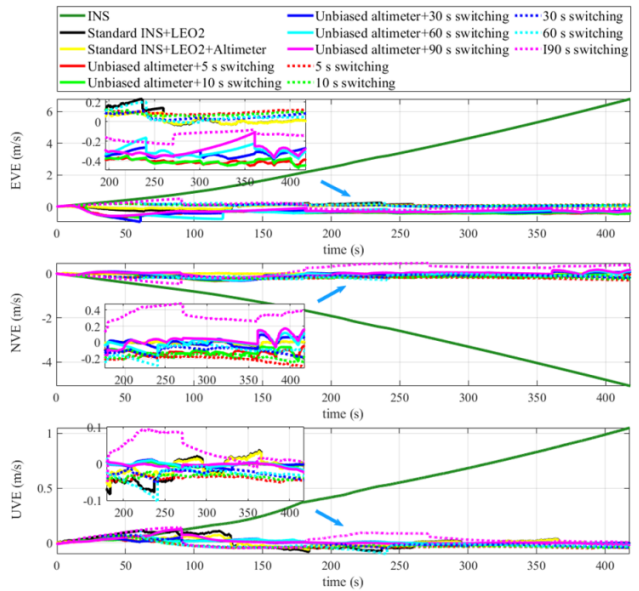

(b) Velocity error curve

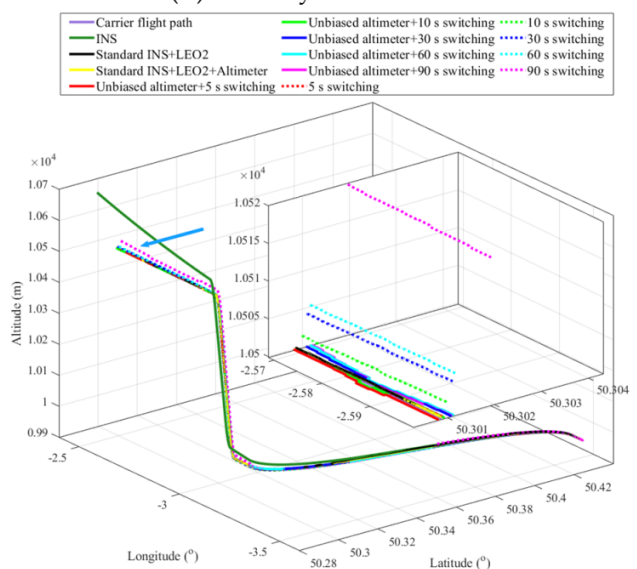

(d) 3-dimensional trajectory error curve

Figure 6. The experimental results of the INS + LEO2 + unbiased altimeter and no altimeter scenarios under adjacent orbits. 


\subsubsection{Comparison of Unbiased Altimeters under the Same and Adjacent Orbit}

To verify the pros and cons of the navigation and positioning algorithms under the same orbit and adjacent orbits after combining the altimeter, we compare the two algorithms by simulation. Figure 8 shows the comparison of the effect of INS + LEO2 switching + unbiased altimeter positioning algorithms under the same orbit and adjacent orbits.

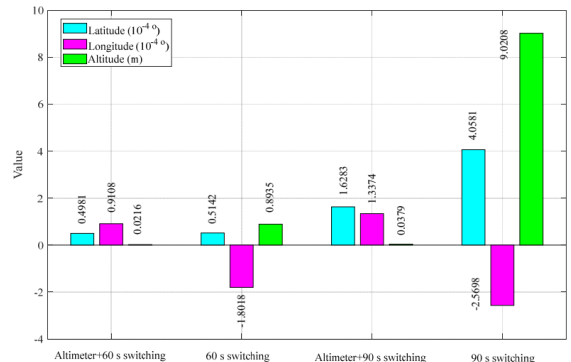

(a) Mean value statistics

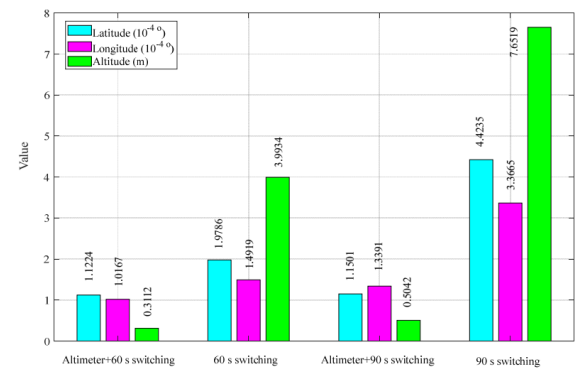

(b) Standard deviation statistics

Figure 7. Under the adjacent orbit, the navigation and positioning error statistics of Table 2. Switching + unbiased altimeter algorithm and the INS + LEO2 switching algorithm when the switching times are 60 and 90 s, respectively.
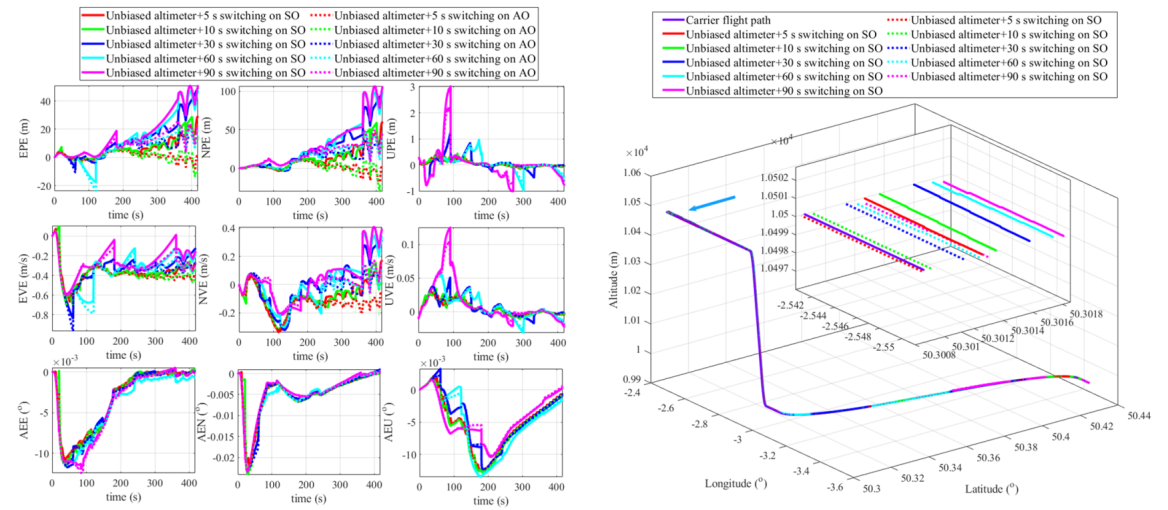

(a) Comparison of position and velocity attitude error curves (b) Comparison of 3-dimensional trajectory error curves

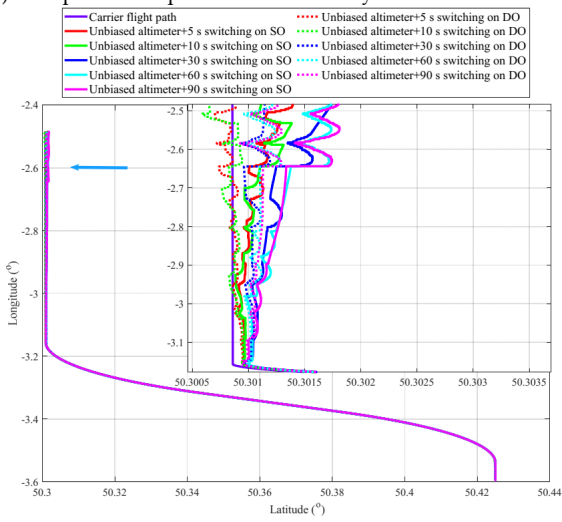

(c) Comparison of 2-dimensional trajectory error curves

Figure 8. Comparison of the effect of INS + LEO2 switching + unbiased altimeter positioning algorithm under the same and adjacent orbits.

From Figure 8 we can see that whether it is position error, or velocity error and attitude error, the effect of INS + LEO2 switching + unbiased altimeter positioning algorithm in adjacent orbits is better than that of the INS + LEO2 switching + unbiased altimeter in the same orbit positioning algorithm; this is also true in terms of trajectory error. We take the statistical results of Figures 5 and 7 as an example: when the switching time is $60 \mathrm{~s}$, compared to the INS + LEO2 switching + unbiased altimeter positioning algorithm under 
the same orbit, in terms of mean statistics, the INS + LEO2 switching + unbiased altimeter positioning algorithm in the errors of longitude, latitude and altitude have improved by $80.38 \%, 49.43 \%$ and $27.52 \%$, respectively, and the corresponding standard deviations have improved by $57.38 \%, 54.65$ and $8.28 \%$, respectively. When the switching time is $90 \mathrm{~s}$, in terms of mean value statistics, the INS + LEO2 switching + unbiased altimeter positioning algorithm under adjacent orbits, the errors of longitude, latitude and altitude have improved by $40.42 \%, 40.07 \%$ and $2.57 \%$, respectively, and the corresponding standard deviations have improved by $56.53,41.99 \%$ and $20.49 \%$, respectively. It can be seen that the use of adjacent orbits is still better than the same orbit, there are similar conclusions at other switching times; due to space limitations, we will not make too many arguments here.

\subsubsection{Comparison of Different Altimeter Deviation Algorithms under Fixed Switching Time}

Altimeters have important applications in some areas of life safety. According to reports, in 2009, Flight TK1951 was caused by the "-8 feet (approximately $-2.4 \mathrm{~m}$ )" accident during the approach phase, which was a disaster that caused the aircraft to crash and die due to inaccurate altimeter readings [40]. Today, such accidents have occurred from time to time; it can be seen that in addition to the operation and control of the crew and air traffic controllers, the accuracy of the altimeter is also a key factor in reducing accidents, therefore, it is necessary for us to carry out qualitative and quantitative analysis on the navigation and positioning error of the algorithm under different altimeter deviations. To explore the impact of different altimeter errors on the navigation and positioning performance, we set the switching time as $5 \mathrm{~s}$, and combined with the accuracy level of the actual altimeter, according to Table 3 , we divide the accuracy of the altimeter into: no deviation, fixed deviation $\pm 5 \mathrm{~m}$, and fixed deviation $\pm 20 \mathrm{~m}$, and then experiment with these different altimeter scenarios. The results are shown in Figure 9. Here, we also include the standard LEO2 switching + INS navigation and positioning algorithm and the standard LEO2 switching + INS + altimeter navigation and positioning algorithm as a reference.

From Figure 9, we can also obtain a similar law, that is: under the same altimeter deviation condition, the navigation and positioning performance of the INS + LEO2 switching navigation positioning algorithm based on adjacent orbits is also better than that of the INS + LEO2 switching navigation positioning algorithm under the same orbit. On the same type of orbit (such as the same or adjacent orbit), as the altimeter deviation increases, the navigation and positioning performance gradually deteriorates. To quantitatively analyze the differences in navigation and positioning under different altimeter scenarios, we have calculated the trajectory errors of the INS + LEO2 switching navigation and positioning algorithm with different altimeter deviations when the fixed switching time is $5 \mathrm{~s}$. The results are shown in Figure 10.

From Figure 10, we can find that under the condition that the altimeter has no deviation, the INS + LEO2 switching navigation and positioning algorithm under the same orbit and the adjacent orbit can reach good location service effects, and the effect under adjacent orbits is better, especially in terms of longitude and latitude performance. However, the difference between the two algorithms in terms of high performance is not large, which is not difficult to understand, since to some extent, when there are only two visible satellites, the difference between the same orbit and the adjacent orbit is not much, but the satellite velocity vectors of the two orbit types are different, and the addition of an altimeter, in the vertical direction, has a similar improvement effect on the INS + LEO2 switching algorithm under the two orbit types. There are similar conclusions in other altimeter deviation cases. It is worth mentioning that if we deduct the fixed height deviation of the navigation positioning algorithm with fixed altimeter deviation, the robustness of the corresponding algorithm is also very good. Taking height deviations of 5 and $20 \mathrm{~m}$ as examples, under the same orbit and adjacent orbit, after deducting the fixed deviation, the absolute value of the mean value of the height difference of the INS + LEO2 switching + altimeter $5 \mathrm{~m}$ deviation algorithm is about $0.03 \mathrm{~m}$, and the absolute value of the mean height difference of the INS + LEO2 switching + altimeter $20 \mathrm{~m}$ deviation algorithm is about $0.2 \mathrm{~m}$. This also requires 
us to choose an altimeter with less deviation as much as possible in practical applications, to improve the accuracy of location services.
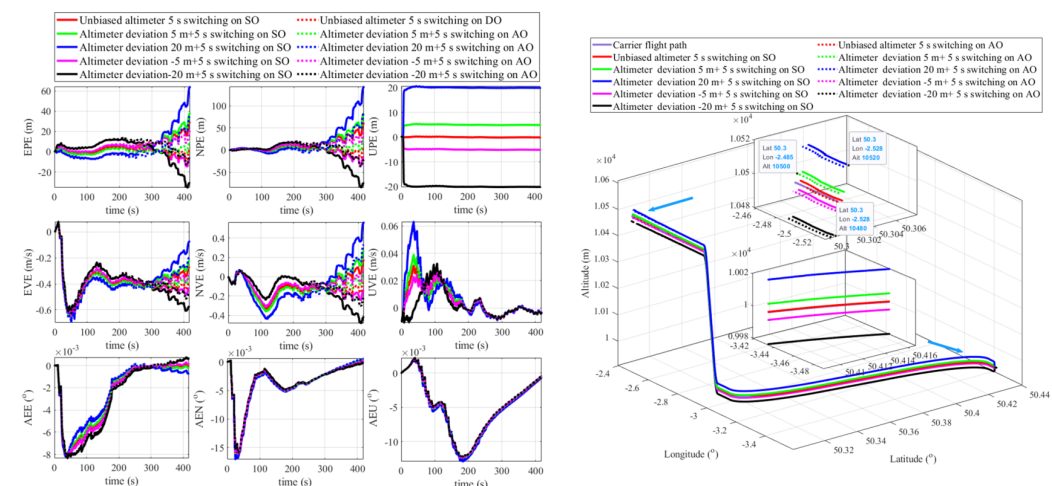

(a) Comparison of position, velocity and attitude error curves (b) Comparison of 3-dimensional trajectory error curves

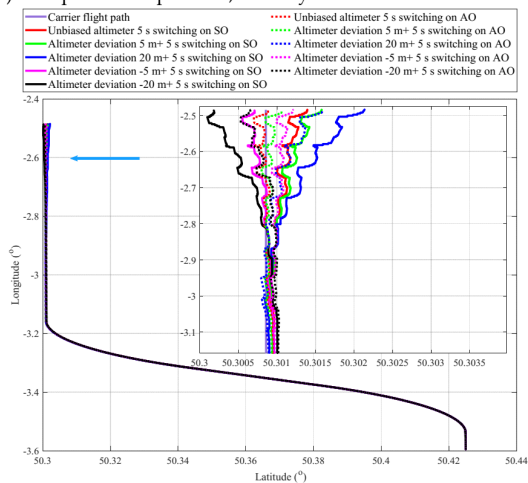

(c) Comparison of 2-dimensional trajectory error curves

Figure 9. Comparison of navigation and positioning of different altimeter deviation scenarios under 5 s switching time.

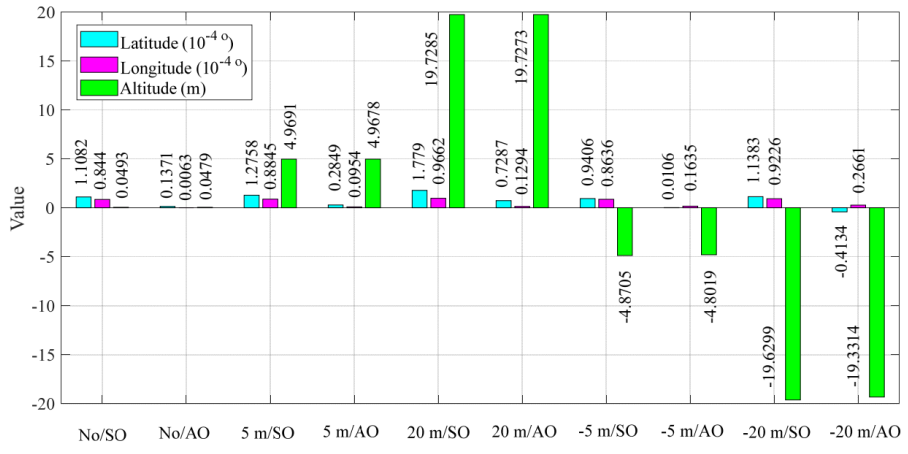

(a) Mean error statistics

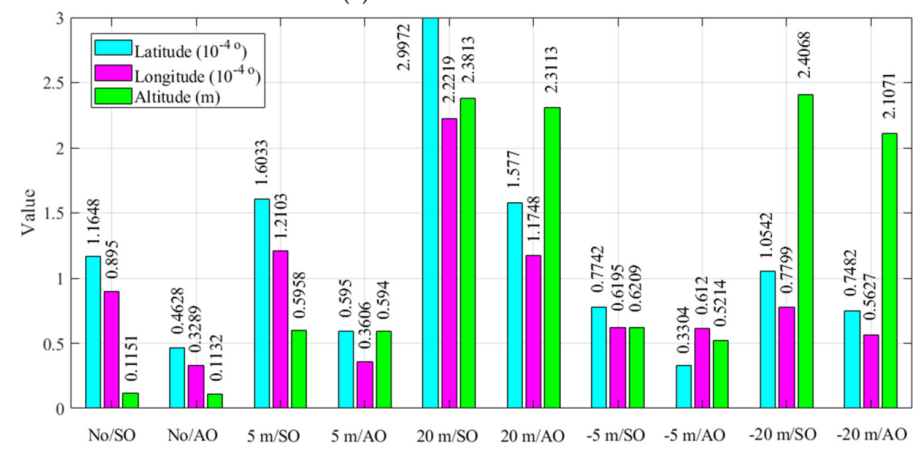

(b) Standard deviation statistics

Figure 10. Navigation and positioning error statistics of different altimeter deviations under a fixed switching time of $5 \mathrm{~s}$. 


\subsubsection{Comparison with Other Algorithms}

To make an objective evaluation of our algorithm, we selected several representative algorithms for comparison, the corresponding algorithms and evaluation indicators are shown in Table 4; among them, our algorithm takes the algorithm under the adjacent orbit as an example. We compare and analyze the corresponding algorithms and indicators, and Reference [20] is also compared based on the switching algorithm under an adjacent orbit.

Table 4. Comparison of different algorithms.

\begin{tabular}{llll}
\hline Error Index & Algorithm & Mean & Standard Deviation \\
\hline \multirow{2}{*}{ Longitude $\left(^{\circ}\right)$} & this paper & $0.1371 \times 10^{-4}$ & $0.4628 \times 10^{-4}$ \\
& Reference [15] & $2.4564 \times 10^{-4}$ & $157.0000 \times 10^{-4}$ \\
& Reference [17] & $/$ & $/$ \\
& Reference [20] & $-0.06539 \times 10^{-4}$ & $0.56238 \times 10^{-4}$ \\
& Reference [21] & $1.6753 \times 10^{-4}$ & $0.1320 \times 10^{-4}$ \\
\hline \multirow{3}{*}{ Latitude $\left(^{\circ}\right)$} & this paper & $0.0063 \times 10^{-4}$ & $0.3289 \times 10^{-4}$ \\
& Reference [15] & $1.7986 \times 10^{-4}$ & $134.0000 \times 10^{-4}$ \\
& Reference [17] & $/$ & $/$ \\
& Reference [20] & $-0.0539 \times 10^{-4}$ & $0.4452 \times 10^{-4}$ \\
& Reference [21] & $16.0000 \times 10^{-4}$ & $3.5382 \times 10^{-4}$ \\
\hline \multirow{2}{*}{ Altitude $(\mathrm{m})$} & this paper & 0.0479 & 0.1132 \\
& Reference [15] & 35.9300 & 14.1989 \\
& Reference [17] & 2.0434 & 0.6221 \\
& Reference [20] & -0.0543 & 0.5229 \\
& Reference [21] & 34.6000 & 6.0518 \\
\hline
\end{tabular}

From Table 4, we can find that our proposed algorithm has greater advantages than the algorithm [15] in terms of longitude, latitude, and altitude indicators, especially in altitude indicators. Compared with the dual-system positioning algorithm proposed by Reference [17], our algorithm also has certain advantages. Compared with the algorithm proposed by Reference [20], although our algorithm is slightly inferior in the mean longitude, the standard of the longitude of our algorithm is $0.4628 \times 10^{-4 \circ}$, which is better than the standard deviation of $0.56238 \times 10^{-4 \circ}$ of the algorithm [20]; this shows that our algorithm has smaller fluctuations in longitude errors and more concentrated errors, and our algorithm has certain advantages in terms of latitude and altitude indicators. Compared with the algorithm proposed by Reference [21], in addition to the longitude and latitude indicators, our algorithm has better accuracy in terms of altitude indicators. From these comparisons, we can see that our proposed algorithm is better than the traditional dual-satellite positioning algorithm and dual-mode system positioning algorithm and even some advanced location service solutions, therefore, our proposed algorithm can be used as a good reference solution in difficult environments.

\section{Conclusions}

We present a giant LEO constellation dual-satellite alternate switching ranging integrated navigation algorithm combined with an altimeter; based on the inherent characteristics of LEO satellites and the advantages of the ELO constellation, the use of LEO constellation for navigation and positioning can eliminate the need to consider clock bias (this is also considerable for reducing the cost of receivers and on-board equipment). In addition, by constructing the position of the moving carrier as a large ellipsoid similar to the earth, the navigation and positioning model can be described more accurately, and then the dynamic navigation and positioning solution can be realized, which has the advantage of accurate positioning. Our experimental results show that only by combining the altimeter, our algorithm can significantly improve the large navigation and positioning error caused by the INS + LEO2 switching without altimeter when the switching time is large. In addition, it is compared with the traditional dual-satellite navigation and 
positioning algorithm, dual-mode system navigation and positioning algorithms and some advanced navigation and positioning algorithms in difficult environments and in those cases it can significantly improve the position error. In practical applications, we can choose an altimeter with different accuracy according to our needs, even if the altimeter has a large deviation, but when we deduct the fixed error of the altimeter, the effect is still obvious. Since the LEO constellation is closer to earth, navigation and positioning based on the LEO constellation will gain many advantages over the $\mathrm{MEO}$ and $\mathrm{HEO}$ constellations. For example, the propagation delay is relatively low, so compared with the MEO and HEO constellations, the real-time performance is stronger; in addition, due to the light weight, small size and low cost of LEO satellites, the constellation can be supplemented by rapid deployment. In general, our algorithm is a high-precision, low-cost, and real-time location service solution, and it is suitable for navigation and positioning in difficult environments such as occlusion, urban blocks, and mountains.

Author Contributions: Y.Y. and L.Y. conceived the conceptualization and algorithm. L.Y. completed the implementation of the algorithm and the writing of the paper, and supported the writing-review and editing. X.J. completed some preliminary simulations, and performed preliminary research and summary of the work. H.L. provided theoretical guidance and suggestions for revision of the paper. H.Y. and Y.X. provided funding support and provided necessary assistance for thesis writing. All authors have read and agreed to the published version of the manuscript.

Funding: Funding was supported by National Key Research and Development Program of China (Grant Nos. 2017YFC1500904 and 2016YFB0501301), the National 973 Program of China (Grant No. 613237201506), the Advance Research Project of Common Technology (No. 41418050201) and Open Research Fund of Southwest China Institute of Electronic Technology (No. H18019).

Institutional Review Board Statement: Not applicable.

Informed Consent Statement: Not applicable.

Data Availability Statement: Not applicable.

Conflicts of Interest: The authors declare no conflict of interest.

\section{References}

1. Hein, G.W. Status, perspectives and trends of satellite navigation. Satell. Navig. 2020, 1, 22. [CrossRef]

2. Barnes, D. GPS status and modernization. In Presentation at Munich Satellite Navigation Summit; Air Force Space Command: Los Angeles, CA, USA, 2019.

3. China Satellite Navigation Office. Development of the BeiDou Navigation Satellite System (Version 4.0); CSNO: Beijing, China, 2019.

4. Zrinjski, M.; Barković, Đ.; Matika, K. Razvoj i Modernizacija GNSS-a. Geod. List 2019, 73, 45-65. Available online: https: // hrcak.srce.hr/218855 (accessed on 25 September 2021).

5. Li, X.; Ma, F.; Li, X.; Lv, H.; Bian, L.; Jiang, Z.; Zhang, X. LEO constellation-augmented multi-GNSS for rapid PPP convergence. J. Geod. 2019, 93, 749-764. [CrossRef]

6. Ye, L.; Yang, Y.; Jing, X.; Ma, J.; Deng, L.; Li, H. Single-Satellite Integrated Navigation Algorithm Based on Broadband LEO Constellation Communication Links. Remote Sens. 2021, 13, 703. [CrossRef]

7. Reid TG, R.; Walter, T.; Enge, P.K.; Lawrence, D.; Cobb, H.S.; Gutt, G.; O'Connor, M.; Whelan, D. Navigation from Low Earth Orbit; John Wiley \& Sons: Hoboken, NJ, USA, 2020. [CrossRef]

8. Reid, T.G.R.; Neish, A.M.; Walter, T.; Enge, P.K. Broadband LEO Constellations for Navigation. J. Inst. Navig. 2018, 65, 205-220. [CrossRef]

9. Reid, T.G.R.; Neish, A.M.; Walter, T.F.; Enge, P.K. Leveraging Commercial Broadband LEO Constellations for Navigation. In Proceedings of the 29th International Technical Meeting of the Satellite Division of the Institute of Navigation (ION GNSS+ 2016), Portland, OR, USA, 12-16 September 2016; pp. 2300-2314.

10. Ma, F.; Zhang, X.; Li, X.; Cheng, J.; Guo, F.; Hu, J.; Pan, L. Hybrid constellation design using a genetic algorithm for a LEO-based navigation augmentation system. GPS Solut. 2020, 24, 62. [CrossRef]

11. Khalife, J.; Neinavaie, M.; Kassas, Z.M. Navigation with differential carrier ohase measurements from megaconstellation LEO satellites. In Proceedings of the 2020 IEEE/ION Position, Location and Navigation Symposium (PLANS), Portland, OR, USA, 20-23 April 2020; pp. 1393-1404.

12. Nardin, A.; Dovis, F.; Fraire, J.A. Empowering the Tracking Performance of LEO PNT by Means of Meta-Signals. In Proceedings of the 2020 IEEE International Conference on Wireless for Space and Extreme Environments (WiSEE), Vicenza, Italy, 23 November 2020; pp. 153-158. [CrossRef] 
13. Lai, J.; Liu, J.; Lin, X.; Xiong, Z. Research on Realization of Filtering Method in Integrated Navigation Based on Bei-Dou Double Star Positioning System. J. Astronaut. 2005, 26, 107-111.

14. Huazhang, L.; Yong, C.; Xueyuan, L. Simulation and Analysis Study on the Position Error of Double-Star Position System. In Proceedings of the Multiconference on Computational Engineering in Systems Applications, Beijing, China, 4-6 October 2006; pp. 776-779. [CrossRef]

15. Qi, H.; Shuang, X.; Chen, Y.; Dai, J. Passive Positioning Algorithm Based on Beidou Double-Star. In Proceedings of the 2006 1st International Symposium on Systems and Control in Aerospace and Astronautics, Harbin, China, 19-21 January 2006; pp. 4-582. [CrossRef]

16. Lin, X. A Position Solution Method for Double-Star Position System. Geomat. Inf. Sci. Wuhan Univ. $2009,34,564-567$.

17. Hou, B.; Zhang, X. A Dual-Satellite GNSS Positioning Algorithm of High Accuracy in Incomplete Condition. China Commun. 2016, 13, 58-68. [CrossRef]

18. Tanaka, T.; Ebinuma, T.; Nakasuka, S. Dual-Satellite Lunar Global Navigation System Using Multi-Epoch Double-Differenced Pseudorange Observations. Aerospace 2020, 7, 122. [CrossRef]

19. Hsu, W.; Jan, S. Assessment of using Doppler shift of LEO satellites to aid GPS positioning. In Proceedings of the 2014 IEEE/ION Position Location and Navigation Symposium-PLANS 2014, Monterey, CA, USA, 5-8 May 2014; pp. $1155-1161$.

20. Ye, L.; Yang, Y.; Jing, X.; Li, H.; Yang, H.; Xia, Y. Dual-statellite Alternate Switching Ranging/INS Integrated Navigation Algorithm for Broadband LEO Constellation Independent of Altimeter and Continuous Observation. Remote Sens. 2021, 13, 3312. [CrossRef]

21. Wang, W.; Zhang, H.; Wu, P. Discontinuous Pseudo Range Constrained Inertial/Double Satellites Integrated Positioning. Electron. Opt. Control. 2016, 23, 6-9, 22.

22. Groves, P.; Jiang, Z. Height Aiding, C/N0 Weighting and Consistency Checking for GNSS NLOS and Multipath Mitigation in Urban Areas. J. Navig. 2013, 66, 653-669. [CrossRef]

23. Kubrak, D.; Macabiau, C.; Monnerat, M. Performance analysis of MEMS based Pedestrian Navigation Systems. In Proceedings of the International Technical Meeting of the Satellite Division of the Institute of Navigation, Long Beach, CA, USA, 13-16 September 2005; pp. 2976-2986.

24. Groves, P.D. Principles of GNSS, Inertial, and Multisensor Integrated Navigation Systems, 2nd ed.; Artech House: Fitchburg, MA, USA, 2012; 776p, ISBN 978-1-60807-005-3.

25. Groves, P.D. Principles of GNSS, Inertial, and Multisensor Integrated Navigation Systems; Artech House: Fitchburg, MA, USA, 2008; 503p, ISBN 978-1-58053-255-6.

26. Julier, S.J. The scaled unscented transformation. In Proceedings of the American Control Conference, Anchorage, AK, USA, 8-10 May 2002; Volume 6, pp. 4555-4559. [CrossRef]

27. Julier, S.J. Unscented filtering and nonlinear estimation. Proc. IEEE 2004, 92, 401-422. [CrossRef]

28. Reali, F.; Palmerini, G. Estimate Problems for Satellite Clusters. In Proceedings of the 2008 IEEE Aerospace Conference, Big Sky, MT, USA, 1-8 March 2008; pp. 1-18. [CrossRef]

29. Krauter, A. Role of the Geometry in GPS Positioning. Period. Polytech. Civ. Eng. 1999, 43, 43-53.

30. Montenbruck, O.; Steigenberger, P. André Hauschild. Multi-GNSS Signal-in-Space Range Error Assessment-Methodology and Results. Adv. Space Res. 2018, 61, 3020-3038. [CrossRef]

31. Montenbruck, O.; Steigenberger, P.; Hauschild, A. Broadcast versus precise ephemerides: A multi-GNSS perspective. GPS Solut. 2015, 19, 321-333. [CrossRef]

32. Groves, P.D.; Adjrad, M. Likelihood-based GNSS positioning using LOS/NLOS predictions from 3D mapping and pseudoranges. GPS Solut. 2017, 21, 1805-1816. [CrossRef]

33. Groves, P.D. GNSS Solutions: Multipath vs. NLOS signals. How Does Non-Line-of-Sight Reception Differ From Multipath Interference. Inside GNSS Mag. 2013, 8, 40-42.

34. Ye, L.; Fan, Z.; Zhang, H.; Liu, Y.; Wu, W.; Hu, Y. Analysis of GNSS Signal Code Tracking Accuracy under Gauss Interference. Comput. Sci. 2020, 47, 245-251.

35. Lvyang, Y.E. Simulation Generate and Performance Analyse on BDS-3 B1C Signal; University of Chinese Academy of Sciences: Beijing, China, 2019.

36. Xue, L.; Wu, W.; Li, X. A New Very Long Baseline Interferometry Method Based on the Uplink Signal. IEEE Access 2020, 8 , 75833-75845. [CrossRef]

37. Xue, L.; Wu, W.; Li, X.; Yang, Y. Design of Tracking, Telemetry, Command (TT\&C) and Data Transmission Integrated Signal in TDD Mode. Remote Sens. 2020, 60, 4573-4582.

38. Del, P.I.; Cameron, B.G.; Crawley, E.F. A technical comparison of three low earth orbit satellite constellation systems to provide global broadband. Acta Astronaut. 2019, 159, 123-135.

39. Groves, P.D. Principles of GNSS, inertial, and multisensor integrated navigation systems. Ind. Robot 2013, 67, 191-192.

40. Miltner, M.; Duan, P.P.; Haag, M. Modeling and utilization of synthetic data for improved automation and human-machine interface continuity. In Proceedings of the 2014 IEEE/AIAA 33rd Digital Avionics Systems Conference (DASC), Colorado Springs, CO, USA, 5-9 October 2014; pp. 2D4-1-2D4-14. [CrossRef] 